\title{
A miniaturized sensing device with ionically imprinted nanostructured films for the ultrasensitive detection of ions
}

\author{
Antonio Ruiz ( $\sim$ a.gonzalez.16@ucl.ac.uk) \\ University College London, Institute for Materials Discovery \\ Xun Cao \\ Nanyang Technological University https://orcid.org/0000-0002-6034-381X \\ Yizhong Huang \\ Nanyang Technological University \\ Kwang-Leong Choy \\ University College London, Institute for Materials Discovery
}

\section{Article}

Keywords: Ion imprinting, nanoporous silica, ion sensor

Posted Date: February 15th, 2021

DOI: https://doi.org/10.21203/rs.3.rs-216530/v1

License: (c) (i) This work is licensed under a Creative Commons Attribution 4.0 International License.

Read Full License 


\title{
A miniaturized sensing device with ionically imprinted nanostructured films for the
}

\section{ultrasensitive detection of ions}

\author{
Antonio Ruiz-Gonzalez ${ }^{a}$, Cao Xun ${ }^{b}$, Huang Yizhong ${ }^{b}$, and Kwang Leong Choy, ${ }^{a,}$
}

a. Institute for Materials Discovery, University College London, London, WC1E 7JE United Kingdom.

b. School of Materials Science and Engineering, Nanyang Technological University, 50 Nanyang Ave, Singapore 639798

Keywords: Ion imprinting, nanoporous silica, ion sensor

\section{Abstract}

The detection of ions is essential for a wide range of applications including biomedical diagnosis, and environmental monitoring among others. However, current ion sensors are based on thick sensing films (typically $100 \mu \mathrm{m}$ ), requiring time-consuming preparations, and have a thermodynamic limit to their sensitivity of $59 \mathrm{mV} \cdot \log [\mathrm{C}]^{-1}$. Such configuration hinders the development of high-performance ion sensors due to the inherent limitations of the bulk diffusion of ions inside sensors. Consequently, they cannot be applied for highprecision applications that require high sensitivity. Furthermore, the research of anion monitoring is hampered due to the limited availability of molecular receptors with acceptable performances. We overcome such limitations by using a $300 \mathrm{~nm}$ nanostructured sensing film based on a novel nanoporous ion imprinted core-shell silica/gold nanoparticulate sensing film. The novel sensing film was highly selective towards chloride ions $\mathrm{w}^{1}$ hen compared to other

\footnotetext{
$1 *$ Corresponding author:

k.choy@ucl.ac.uk
} 
anions such as nitrate, sulphate and carbonate. Moreover, this nanostructured sensing film exhibited above 3 -fold higher sensitivity $\left(-186.4 \mathrm{mV} \cdot \log [\mathrm{C}]^{-1}\right)$ towards chloride ions when compared to commercial devices. Such breakthrough has led to the fabrication of the smallest and most sensitive reported anion sensor working on open circuit potentiometry, with an exceptional selectivity towards chloride ions.

\section{Introduction}

The measurement and monitoring of ions in solution is essential for a wide range of applications such as the clinical analysis, physiological monitoring ${ }^{[1-3]}$, water quality evaluation $^{[4]}$ performance monitoring of energy storage devices ${ }^{[5]}$, corrosion monitoring in structural engineering ${ }^{[6]}$, or agricultural smart farming ${ }^{[7]}$ among others. Specifically, anions imbalance play a key role in the development of multiple diseases such as chronic kidney disease ${ }^{[8]}$ and can lead to multiple pathological conditions such as acidosis, caused by an accumulation of acid that can be a consequence of an excess of chloride ions, with deleterious effects in the body ${ }^{[9]}$. In addition, excessive presence of anions such chloride ions in aquatic environments can lead to the acidification of the environment or decrease the anti-microbial capability of treated water. Therefore, its concentration must be tightly regulated ${ }^{[10]}$. From an industrial perspective, the presence of chloride ions is a strong indicator of environmental degradation processes such as corrosion ${ }^{[6]}$ and the quality and health of the structural engineering materials ${ }^{[11]}$. However, due to the generally small size of these anions, less than $0.2 \mathrm{~nm}$ in the case of chloride ion $\left(\mathrm{Cl}^{-}\right)$, and their scarce lipophilicity ${ }^{[12]}$, only a few commercial anion sensors available in the market, and there are limited reported literature on these sensors. Most of these sensors also exhibit a rather poor performance due to ionic interferences and hence low selectivity ${ }^{[13]}$.

While there is a demand for miniaturized anion sensing devices with super-sensitivity, greater than the Nernst limit of $59 \mathrm{mV} \cdot \log [\mathrm{C}]^{-1}$ for the measurement of anions for high-precision 
applications, to date, no approaches have been able to fulfil such requirements. Traditionally, the main technology for the development of ion-selective electrodes consists of a plasticized PVC membrane with embedded ionophores, which are specific complexing agents that perform the electrolyte recognition process ${ }^{[14]}$. However, numerous studies have been focused on the formalization of ion-selective electrodes theory ${ }^{[15-18]}$, and concluded that the sensitivity of the devices is thermodynamically limited to $59 \mathrm{mV} \cdot \log [\mathrm{C}]^{-1}{ }^{[14]}$ In addition, their miniaturization has been proven to be challenging, and typical sensors tend to be thick, with a typical thickness in the range of $100 \mu \mathrm{m}^{[19]}$.

Early attempts in the miniaturization of these membranes showed a faster ion equilibration kinetics when they were reduced to $35 \mu \mathrm{m}$ thick layers ${ }^{[20]}$. However, no sub-micron level films operating at zero-current conditions have been reported in the literature. Such level of reduction could offer advantages in terms of the time-response of the sensors and allow a better integration in miniaturized systems, especially desirable for portable or wearable technologies. As such, an enhancement in the sensitivity and miniaturization of the ion sensors would be beneficial for portable and wearable applications. Such miniaturization of the sensing materials would also be beneficial to interface this sensor technology with upcoming technologies such as ion-selective field effect transistors ${ }^{[21]}$. In addition, the development of rapid sensors operating with super-sensitivity beyond the Nernst limit is crucial to characterize the concentration of ions locally around neurons, which could help in the diagnosis of seizures ${ }^{[22]}$, and could allow the genome sequencing ${ }^{[23]}$.

Thus far, the performance of commercial and state-of-the-art ion sensors based on ionophorecontaining polymeric films are constrained to the use of relatively thick sensing films, in the range of micrometers, and a restricted sensitivity of $59 \mathrm{mV} \cdot \log [\mathrm{C}]^{-1}$. Shklovskii et al. ${ }^{[24,25]}$ observed that this sensitivity limit could be surpassed when charged surfaces are employed, 
offering the possibility of developing a new family of sensors with super-Nernstian sensitivities. This observation was a consequence of the strong interaction between the opposite charges of ions and the membranes. Although this work was based on the study of macroions, recently Sivakumarasamy et al. ${ }^{[26]}$ demonstrated the suitability of this concept to develop an ion-sensor based on specific sites on a $25 \mathrm{~nm}$ 0D transistor using a silica surface for the simultaneous detection of multiple cations in serum. However, the costly fabrication methods, based on nanolithography and their sensing mechanism, requiring complex nanofluidic channels, led to challenging data analysis, due to the necessity of modelling the response of the sensor in the presence of multiple electrolytes. This has not only led to a large uncertainty on the measurements and sensing data but also makes them an impracticable technology commercially. Moreover, this device configuration could not be used for the determination of negatively charged ions such as chloride ions. Since then, multiple ionic exchange resins have been developed for the adsorption of multiple ions, limited to cations such as zinc ${ }^{[27]}$, nickel ${ }^{[28]}$ and lead ${ }^{[29,30]}$. However, these materials lacked specificity, limiting their suitability as components of ion sensors, and in general there is a lack of advances in the field of anion-selective materials. In fact, the discovery of anions sensitive materials or materials for the selective detection of certain anions represents a major challenge in analytical chemistry, electrolyte monitoring in patients, diagnosis, and anion measurement for environmental evaluation.

The exploitation of imprinted technology for the fabrication of sensing materials represents a promising alternative in this field, allowing the development of tailor-made sensing electrodes with reduced dimensions. The material imprinting process is based on the synthesis of sensitive species by direct polymerization of functional monomers in the presence of a template. Thus, specific recognition elements can be developed virtually for any compound ${ }^{[31,}$ ${ }^{32]}$. To date, this approach has been applied mostly to the detection of cations, including 
copper $^{[33-35]}$, cadmium ${ }^{[36,37]}$, mercury ${ }^{[38]}$ and lead ${ }^{[39]}$, and rare earth metal ions such as europium $^{[40]}$ and yttrium ${ }^{[41]}$. However, due to the weak interaction of the anions with the chelating species commonly found, thus far there has been a limited development in this field. Although similar imprinting approaches have been applied to the fabrication of sensing materials for the detection of relatively small anions such as phosphate and nitrate ions ${ }^{[42,43]}$ for potential applications in environmental monitoring, its potential for the specific interaction with single anions such as chloride ions has yet to be explored.

Within the present work, a miniaturized device with a nanometer-size thick sensing film was developed by exploiting the ion imprinting technology with a novel material functionalization and grafting methods. The new device structure reported here is based on the use of a porous core-shell configuration with gold nanoaggregates and a shell of nanoporous silica containing positively charged amine groups, which enhanced the sensitivity of the sensors and greatly reduced the preparation time. These Au nanoparticles were synthesized to be specific towards chloride by condensing silica monomers in the presence of $\mathrm{HCl}$, and using gold nanoaggregates as the core material. Such material transformed the typical signal transduction mechanism of the traditional sensors based on the bulk diffusion of electrolytes, into a surface effect through the adsorption of ions. Such novel materials and sensing device configuration reported here enabled the development of the smallest Ion sensor working on Open Circuit potential with an unprecedented sensitivity and selectivity.

\section{Results and discussion}

\section{Structure of the nanostructured sensing layer}

The first step in the development of the nanostructured sensors was the characterization of the fabricated gold nanoclusters. Such gold nanoclusters were synthesized by a simple reduction method using citric acid. Gold was used for the transduction of the electrochemical signal due 
to its strong interaction with chloride ions ${ }^{[44]}$ and its low charge transfer resistance ${ }^{[45]}$. A solid shell of nanoporous silica using tetraethyl orthosilicate (TEOS) as a precursor was then integrated, enabling the adsorption, increasing the specificity of the sensor, and allowing the attachment to the gold contact. In addition, the amine group present in aminopropyl triethoxysilane (APTES) was used as the recognition element towards chloride ion species. This functional group was proven to be versatile, enhancing the interaction with the anions after being positively charged using an acidic solution of $\mathrm{HCl}$.

The specificity of this material was accomplished by the synthesis of the TEOS and gold precursors in the presence of $\mathrm{HCl}$, which contains the target ion $\mathrm{Cl}^{-}$, allowing the formation of a specific pore structure in this material. The structure of this recognition element, using a porous silica as the receptor located on the outer shell of the sensor, was confirmed by high resolution transmission electron microscopy (HRTEM) (Figure 1.a) and Figure 1.b)). Most of the gold particles used as the core were formed along the zone axis of [ $\left[\begin{array}{lll}1 & 0 & 1\end{array}\right]$, with [ $\left[\begin{array}{lll}1 & 1 & 1\end{array}\right]$ being the strongest crystallographic orientation (Figure 1.c)), and the distribution of both gold and silica could be observed using the Z-contrast (Figure 1.d) and Figure 1.e)). Both elements could be observed together within the core-shell structure of the ionically imprinted gold/silica nanoparticles (Figure S.1). The synthesized gold nanoclusters were homogeneous in size, typically in the range of $5 \mathrm{~nm}$, and they were crystalline as confirmed by the strong diffraction spots in the Fast Fourier Transform (FF) pattern. 
a)

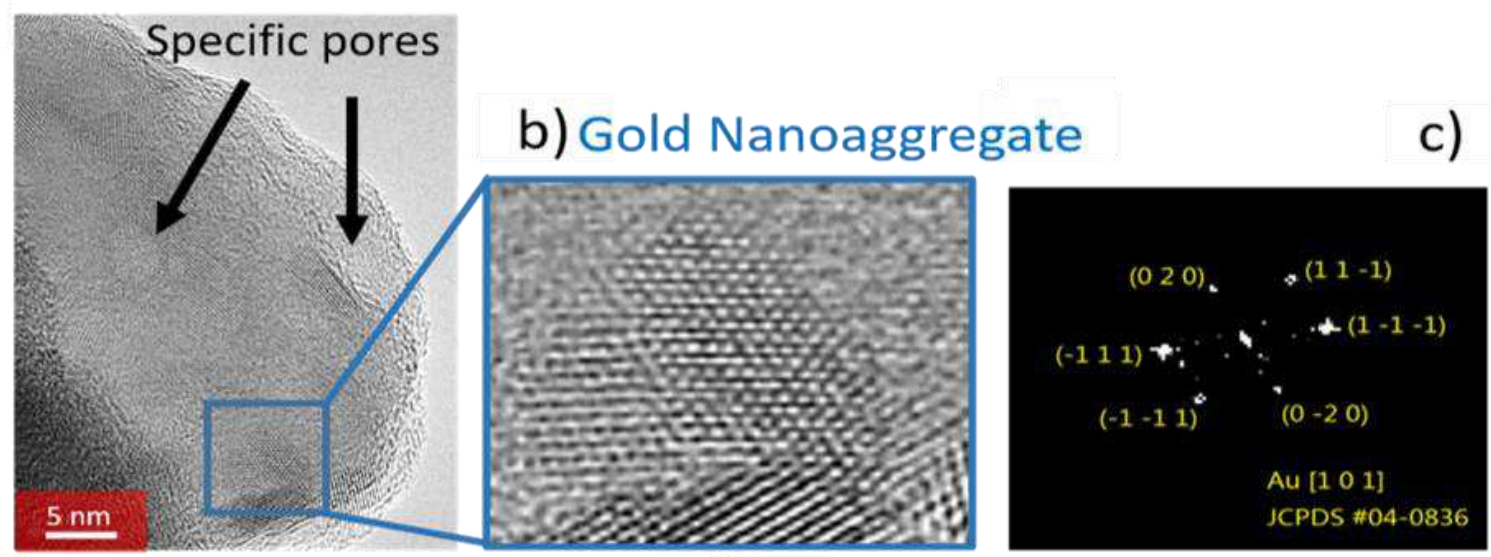

d)

e)
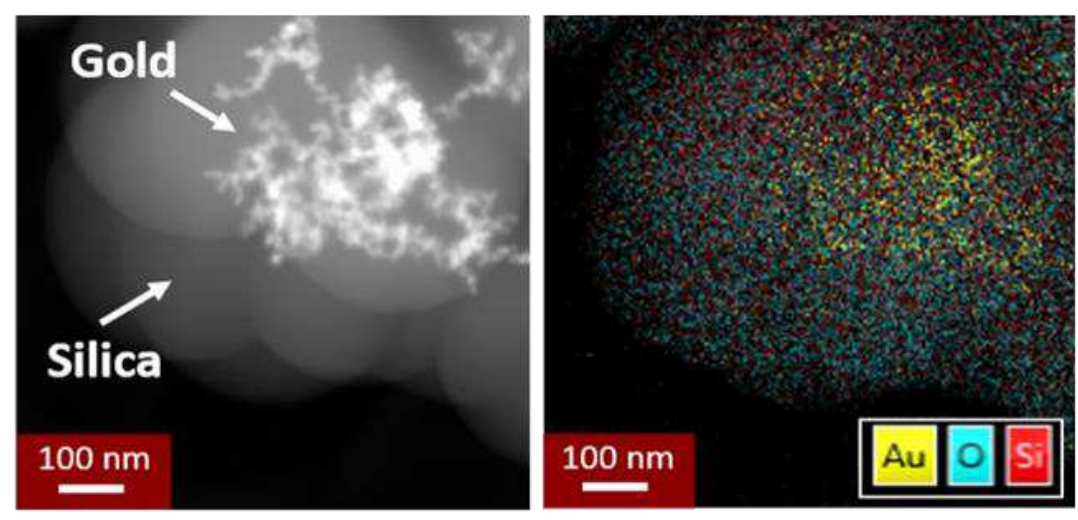

Figure 1. HRTEM visualization of the silica-Au core-shell structure. a) Detail of the surface of the ionically imprinted silica onto the gold nanoclusters where the pore structure is highlighted with pore diameter typically around $3 \mathrm{~nm}$. b) A gold nanoaggregate is magnified, allowing the visualization of the crystalline structure. c) FFT diffraction pattern showing the presence of gold with [ $\left[\begin{array}{lll}1 & 1 & 1\end{array}\right]$ as the preferred orientation. d) HAADF-STEM revealing the distribution of both silicon and gold elements across the core-shell structure using a $\mathrm{Z}$ contrast. e) EDX mapping of $\mathrm{Au}$ (yellow) and $\mathrm{Si}$ (Pink) indicating the distribution of both elements within the ionically imprinted Gold/silica nanoparticles.

A porous structure was formed within the ionic imprinted silica/gold, which was consistent with the previous work in silica-based nanoimprinted particles ${ }^{[46]}$. The pore radius here observed was confirmed by BET, being in the range of $1.4 \mathrm{~nm}$ (Figure 2.a)), and a total 
surface area of $108 \mathrm{~m}^{2} \mathrm{~g}^{-1}$. Such surface area was calculated from the isotherms obtained using BET and the associated BET equation (Figure S.2). This pore size is comparable to previous ion imprinted systems based on silica nanoparticles for dissolved metals ${ }^{[47,48]}$.

The spherical architecture of the ionically imprinted nanoparticles with a size in the range of $118 \pm 29 \mathrm{~nm}$ after the synthesis was further corroborated by SEM (Figure 2.b) and Figure S.3). The nanoparticle grafting on the gold electrodes was then carried out by a directional attachment of APTES via EDC/NHS chemistry. A successful anchoring of molecularly imprinted polymeric nanoparticles has been achieved in the literature by previous functionalization with primary amines and posterior reaction with the substrates ${ }^{[49]}$. However, this process had to be modified in our nanoimprinted material since the direct use of the EDC reagent could modify the amine groups present in the specific recognition sites, leading to a loss in sensitivity. Thus a novel fabrication process was developed. Here, the gold electrodes were first functionalized using a self-assembled monolayer of 16-mercaptohexadecanoic acid. A directional attachment of APTES using the standard 1-Ethyl-3-(3dimethylaminopropyl)carbodiimide and N-Hydroxysuccinimide (EDC/NHS) chemistry was then employed, grafting the molecules and exposing the silane groups that could interact with the nanoparticles. Finally, the nanoparticles were deposited. The chemical characterization of the nanoimprinted material was performed using FTIR (Figure 2.c)) which revealed the typical spectrum of APTES, with a peak in the $1390 \mathrm{~cm}^{-1}$ range, indicative of the $-\mathrm{CN}$ stretch. The 1032 and $778 \mathrm{~cm}^{-1}$ peaks were also detected due to the presence of the Si-O-Si structure of silica $^{[50]}$. This 2-step approach for the fabrication of the sensing devices could be applied to the production of an array of up to 5 electrodes onto a glass substrate (Figure 2.d)). 

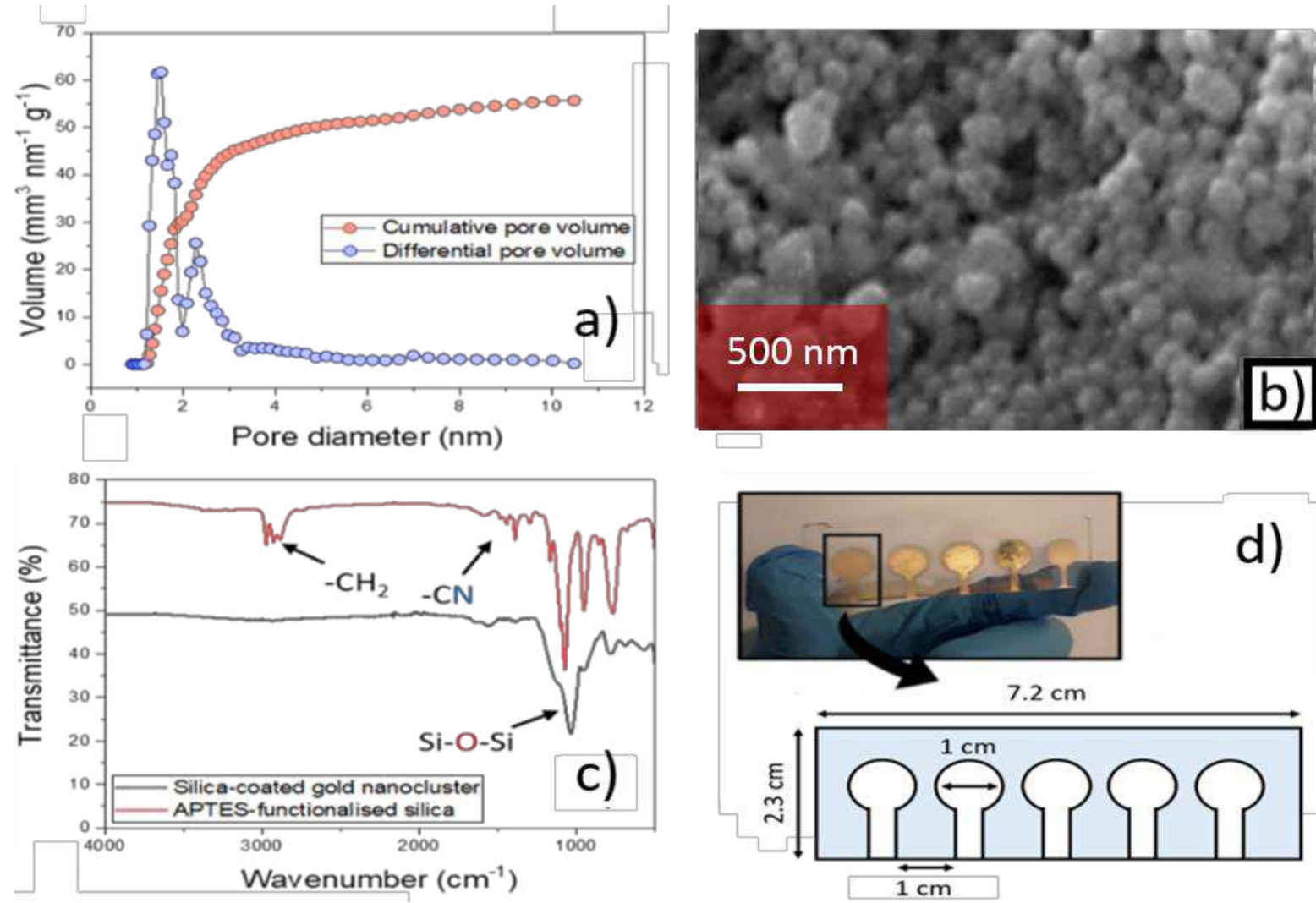

Figure 2. a) Cumulative and differential pore volume obtained by BET, showing a higher presence of pores in the $2.8 \mathrm{~nm}$ range. b) SEM image of the silica encapsulated gold nanoparticles. c) FTIR spectrum of the silica-encapsulated gold when compared with the one obtained for APTES-functionalized silica. The representative peaks of the Si-O-Si bonds present in silicon dioxide $\left(1032\right.$ and $\left.778 \mathrm{~cm}^{-1}\right)$ and APTES $\left(-\mathrm{CH}_{2}\right.$ at $3000 \mathrm{~cm}^{-1}$ and $-\mathrm{CN}$ in the range of $1600 \mathrm{~cm}^{-1}$ ) are highlighted. d) Picture of the thin film sensing device containing the grafted ionically imprinted silica nanoparticles on the surface of gold electrodes. The dimensions of the sensors are highlighted.

\section{Demonstration of the sensing performance of the ultra-thin Imprinted ion sensor}

After grafting the nanoparticles onto $50 \mathrm{~nm}$ thick gold electrodes with an APTES linker, a $290 \mathrm{~nm}$ thick nanostructured film was obtained as measured by a stylus profilometer (Figure 
S.4). To the best of our knowledge, this is the smallest reported potentiometric ion sensor based on Open Circuit Potential (OCP) readings. After the fabrication of the full device, a homogeneous film was obtained, with the presence of regularly distributed domains rich in ion imprinted nanoparticles containing gold. This surface morphology of the film consisting of ion imprinted nanoparticles, formed small aggregates in the range of $500 \mathrm{~nm}$ as observed by SEM equipped with EDS elemental mapping (Figure 3.a)).

The attachment of the ion imprinted nanoparticles to the electrodes using the covalent bond with the APTES linker here reported was analyzed by quartz microbalance. This technique can be used for the determination of weight changes over an electrode in the nanogram range, confirming the success of the functionalization due to the changes in the mass on the electrodes in Figure S.5)). Within the first step of the fabrication, where the APTES group was directionally grafted after the deposition of the self-assembled film, a gain of $188 \pm 20$ ng. $\mathrm{cm}^{-1}$ was observed. The post-modification with silica/gold nanoparticles increased the total weight by $294 \pm 3 \mathrm{ng} . \mathrm{cm}^{-1}$, being consistent with the two-step approach for the nanofilm fabrication. This technique could additionally be employed for the determination of the total amount of adsorbed chloride ions on the surface of the ion imprinted porous silica/gold nanoparticles. Such test was used to confirm the relevance of using the chloride ion templates during the synthesis. In this case, the differences in the mass of the sensors during the conditioning in DI and after their immersion in $0.1 \mathrm{M} \mathrm{KCl}$ solution was recorded, and normalized by dividing the result by the initial weight of the films in DI water. Here, an absorption ratio of $8.1 \Delta \mathrm{ng}_{\mathrm{ion}} . \mathrm{ng}_{\mathrm{eq}}{ }^{-1}$ was obtained when templated gold/silica ionically imprinted nanoparticles were employed. On the contrary, when no $\mathrm{HCl}$ was employed, also called non-templated ion imprinting nanoparticles, a significantly lower absorption with a maximum of $3.4 \Delta \mathrm{ng}_{\text {ion. }} \cdot \mathrm{ng}_{\mathrm{eq}}{ }^{-1}$ was obtained as shown in Figure 3.b). Consequently, these devices could not be employed for the sensing. Such lower absorption 
could be attributed to the reduced formation of nanopores and the low presence of charged functional groups.

A final test of the electrochemical performance was subsequently performed on the sensing device. The OCP of the ion sensor was used as the measurement method, similar to the case of the commercial polymeric based sensing films, which tends to require a low energy consumption. Contrary to the standard commercial polymer-based ion-selective sensors, the devices here employed did not require a long pre-conditioning process. The commercial sensors typically need to be subjected to a highly concentrated solution in the range of $0.1 \mathrm{M}$ of the target analyte for at least $24 \mathrm{~h}$ to reach equilibrium before their usage ${ }^{[51]}$. Consequently, they require time-consuming preparation for such preconditioning process. However, the present ion sensing device reported here could reach the equilibrium with the solution shortly after the synthesis, and only 46 mins were required to reach the equilibrium (Figure 3.c)). The signal stability of the ion sensors was in the range of $1.1 \mathrm{mV} \cdot \mathrm{h}^{-1}$, being significantly lower than the standard commercialized devices for anions, in the range of $5 \mathrm{mV}^{[52]}$, and was stable for at least $13 \mathrm{~h}$ under constant contact with the solution. Thus, the ion sensing devices could be applied to the continuous measurement of electrolytes due to the rapid nature of the adsorption reaction, offering a prompt monitoring of analytes.

When using metal salts that contained chloride ions such as $\mathrm{NaCl}, \mathrm{KCl}$ or $\mathrm{CaCl}_{2}$, a superNernstian response in the range of $-186.4 \mathrm{mV} \cdot \log \left[\mathrm{Cl}^{-}\right]^{-1}$ was achieved within the linear range of the plots, with a limit of detection in the range of $10^{-4} \mathrm{M}$ (Figure 3.d)). This value overcomes the traditional Nernst sensitivity limit of $-59 \mathrm{mV} \cdot \log \left[\mathrm{C}^{-}\right]^{-1}$, representing a new record in the performance of these anion sensors, specifically chloride ions. Here, the sensitivity was calculated by measuring the slope of the responses within the linear range of the measurements (Figure 3.e)). These sensors could be re-used, giving a reproducible 
response towards $\mathrm{Cl}^{-}$. This reproducibility of the measurements was additionally proven using 3 different chlorinated salts. The ion sensor showed a similar response when measuring chloride ions from $\mathrm{NaCl}, \mathrm{KCl}$ and $\mathrm{CaCl}_{2}$ as shown in Figure 3.e). Additionally, the ion sensing device showed an unprecedented selectivity towards chloride ions when compared to other halogens such as $\mathrm{F}^{-}, \mathrm{Br}^{-}$and anions like $\mathrm{SO}_{4}{ }^{2-}, \mathrm{NO}_{3}{ }^{-}$, and $\mathrm{HCO}_{3}{ }^{-}$. These electrolytes were specifically tested due to their essential roles in the clinical diagnostic for diseases resulted from electrolytes imbalance ${ }^{[53-55]}$.

A significant reduction in sensitivity was observed when non-chlorinated salts were employed. F- represented the main interference electrolyte, since the sensors showed a sensitivity of $-70 \pm 23 \mathrm{mV} \cdot \log \left[\mathrm{F}^{-}\right]^{-1}$ to this anion, being almost 3 times lower than the sensitivity towards $\mathrm{Cl}^{-}$. In addition, the sensitivity towards $\mathrm{Br}^{-}$and $\mathrm{SO}_{4}{ }^{2-}$ ions was about 5 times lower than the sensitivity towards $\mathrm{Cl}^{-}$, with $-43 \pm 6 \mathrm{mV} \cdot \log \left[\mathrm{Br}^{-}\right]^{-1}$ and $-40 \pm 7$ $\mathrm{mV} . \log \left[\mathrm{SO}_{4}{ }^{2-}\right]^{-1}$, respectively. A negligible sensitivity value towards nitrate, carbonate and hydroxyl ions were also observed, with $-28 \pm 11,-10 \pm 20$, and $-8 \pm 3 \mathrm{mV} \cdot \log \left[\mathrm{C}^{-}\right]^{-1}$ respectively. However, in the case of $\mathrm{OH}^{-}$, due to the solubility of the silica compounds in the presence of a high $\mathrm{pH}$, the sensors could only be assessed in a concentration of up to $10^{-2} \mathrm{M}$. The reported selectivity here represents a significant enhancement in sensitivity towards chloride ion by at least 3-fold when compared with the currently available commercial technologies for chloride ions detection.

The sensing devices based on silica/gold ionically imprinted nanoparticles confirming the selectivity of this novel ion sensor in a solution containing a mixture of interfering ions, including the above mentioned $\mathrm{F}^{-}, \mathrm{Br}^{-}, \mathrm{SO}_{4}{ }^{2-}, \mathrm{NO}_{3}{ }^{-}$and $\mathrm{HCO}_{3}{ }^{-}$. Such interfering ions did not significantly change the OCP signal of the devices when $10 \mathrm{mM}$ of each ions were included simultaneously in the tested solution (Figure 3.f). As such, the selectivity of the devices was 
demonstrated. Such selectivity was also evaluated by calculating the selectivity coefficient of the silica/gold ionically imprinted nanoparticles, being higher than the previously reported work in the literature, especially in the case of $\mathrm{Br}^{-}$and $\mathrm{F}^{-}$ions ${ }^{[56]}$ (Table S.1).
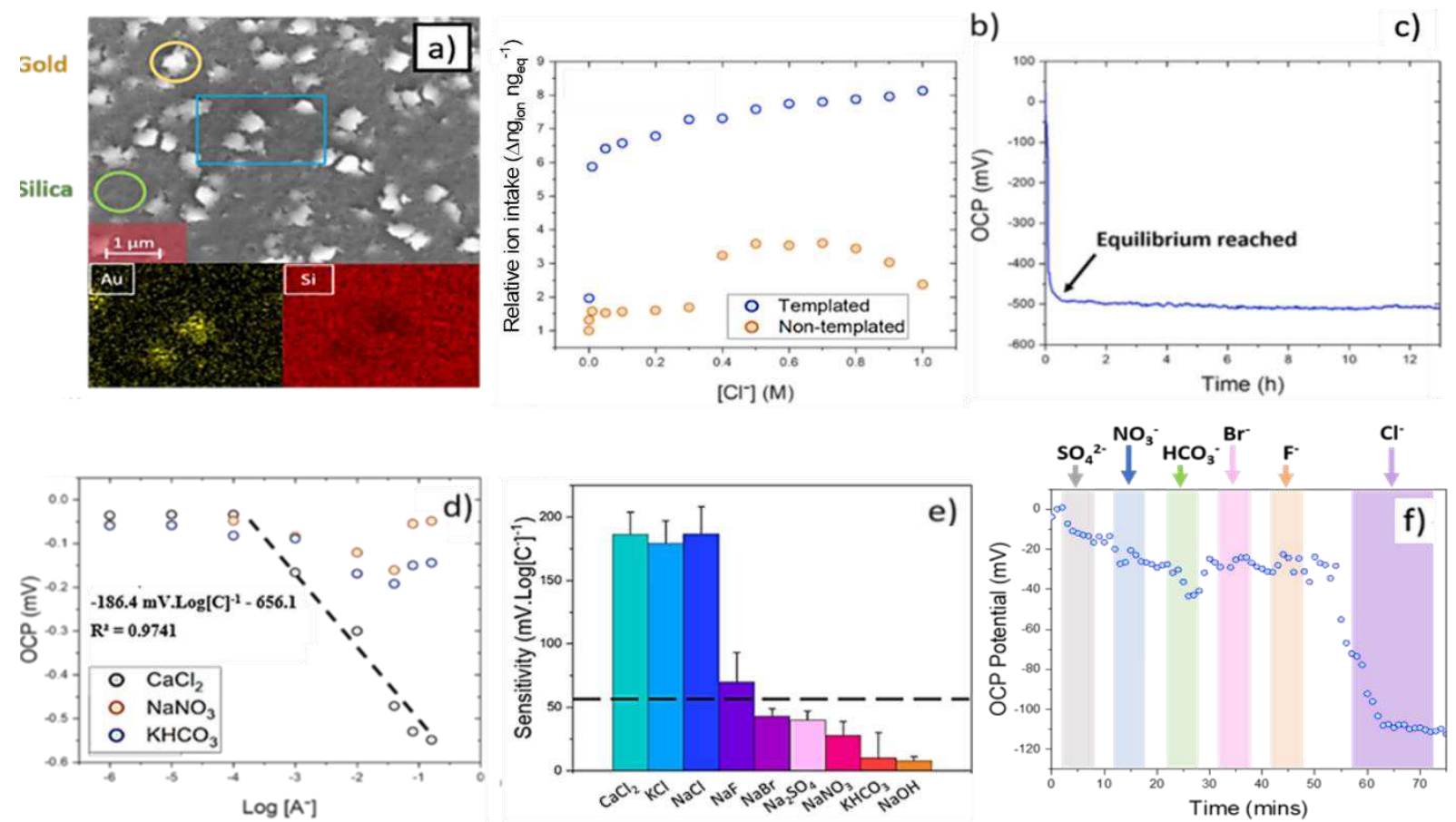

Figure 3. a) SEM image of the sensing film containing the ion imprinted silica/gold nanoparticulate surface morphology. The EDS elemental mapping of gold and silica in the highlighted area is also shown. b) Relative absorption of chloride ions by the ion-imprinted electrodes grafted onto gold surfaces as measured by quartz microbalance. These values were measured by subjecting the film to different concentrations of $\mathrm{NaCl}$, and the results were normalized by the first observed weight value, when only water was used. c) OCP monitoring of the chloride-selective sensors over $13 \mathrm{~h}$ using $\mathrm{KCl}$. The equilibrium point of the sensor in $\mathrm{KCl}$ is indicated. d) Recorded electrochemical potentials at different concentrations of different anions. The calculation of the sensitivity of the devices was exemplified by taking the linear range of the calibration plot. e) Sensitivity profiles of the ionic imprinting-based electrodes after testing with different metal chlorides $\left(\mathrm{NaCl}, \mathrm{KCl}, \mathrm{CaCl}_{2}\right)$ and interference anions from $\mathrm{Na}_{2} \mathrm{SO}_{3}, \mathrm{NaNO}_{3}$ and $\mathrm{KHCO}_{3}$. f) Monitoring of the OCP signal of a sensing device containing the gold/silica ionically imprinted nanoparticles when subjected to a mixed 
concentration of anions. The result of cumulatively adding $10 \mathrm{mM} \mathrm{SO}_{4}{ }^{2-}$ (grey), $10 \mathrm{mM}$ of $\mathrm{NO}^{3-}$ (blue), $10 \mathrm{mM} \mathrm{HCO}_{3}^{-}$(green), $10 \mathrm{mM} \mathrm{Br}^{-}$(pink) and $10 \mathrm{mM} \mathrm{F}^{-}$(orange) was measured. Only a significant change in the potentiometric signal can be observed when $\mathrm{Cl}^{-}$is added.

The amount of template ions $\left(\mathrm{Cl}^{-}\right)$employed during the synthesis of the Ion imprinted silica/gold nanoparticles played a crucial role in the performance of the final device. Since $\mathrm{Cl}^{-}$ was used for the creation of a specific pore by its interaction with the aminated APTES molecules, its concentration and stoichiometry with respect to APTES had to be tightly regulated to ensure a homogeneous formation of the specific pores. This fact was demonstrated using different amounts of $\mathrm{HCl}$ during the synthesis of the ion imprinted silica/gold nanoparticles while keeping the amount of APTES constant and measuring the sensitivity of the devices. When small concentrations of the $\mathrm{HCl}$ template was employed, in the range of $0.5 \mathrm{mM}$, a low sensitivity of $-17.4 \mathrm{mV} \cdot \log \left[\mathrm{Cl}^{-}\right]^{-1}$ was obtained. Such sensitivity was over 10 times lower than the sensitivity achieved in the present work using $1 \mathrm{mM}$ of $\mathrm{HCl}$, which is in the range of $-186.4 \mathrm{mV} \cdot \log \left[\mathrm{Cl}^{-}\right]^{-1}$. Such low sensitivity was attributed to the low availability of the $\mathrm{Cl}^{-}$templates, leading to the formation of non-specific domains on the surface of the nanoparticles since there are APTES molecules that do not interact with the $\mathrm{Cl}^{-}$ templates, and a low degree of quaternisation of such APTES molecules. On the contrary, when higher amounts of $\mathrm{HCl}$ were employed, the sensitivity was still suboptimal, with -99.1 $m V \cdot \log \left[\mathrm{Cl}^{-}\right]^{-1}$. Although such sensitivity was higher than the one achieved when low amounts of $\mathrm{HCl}$ template were employed, it was significantly lower than the optimal sensitivity at $1 \mathrm{mM} \mathrm{HCl}$. Under optimal conditions, the chloride ions were adsorbed by a combination of electrostatic interactions and hydrogen bonds ${ }^{[31]}$. However, when high concentrations of template were used compared to APTES, the pores did not present the 
optimal conditions for such interactions. Consequently, the sensitivity of the devices was reduced (Figure S.6).

\section{Sensing mechanism}

Since our nanostructured sensing film is based on nanoporous core/shell silica/gold nanoparticles, its sensing mechanism is a consequence of the adsorption of ions on the surface of the nanomaterials. This mechanism has been proven by multiple simulations studied on molecularly imprinted materials. Here, this particular molecular structure, forming a specific pore with a specific disposition of functional groups and ligands, in combination with the stoichiometry employed, allowed the formation of a highly sensitive and selective sensing film. The ions present in solution in a solvated form, surrounded by a water shell (Figure 4.a)) can then interact with the amine groups at the surface of the nanoporous core/shell silica/gold nanoparticles (Figure 4.b) and Figure 4c)). 


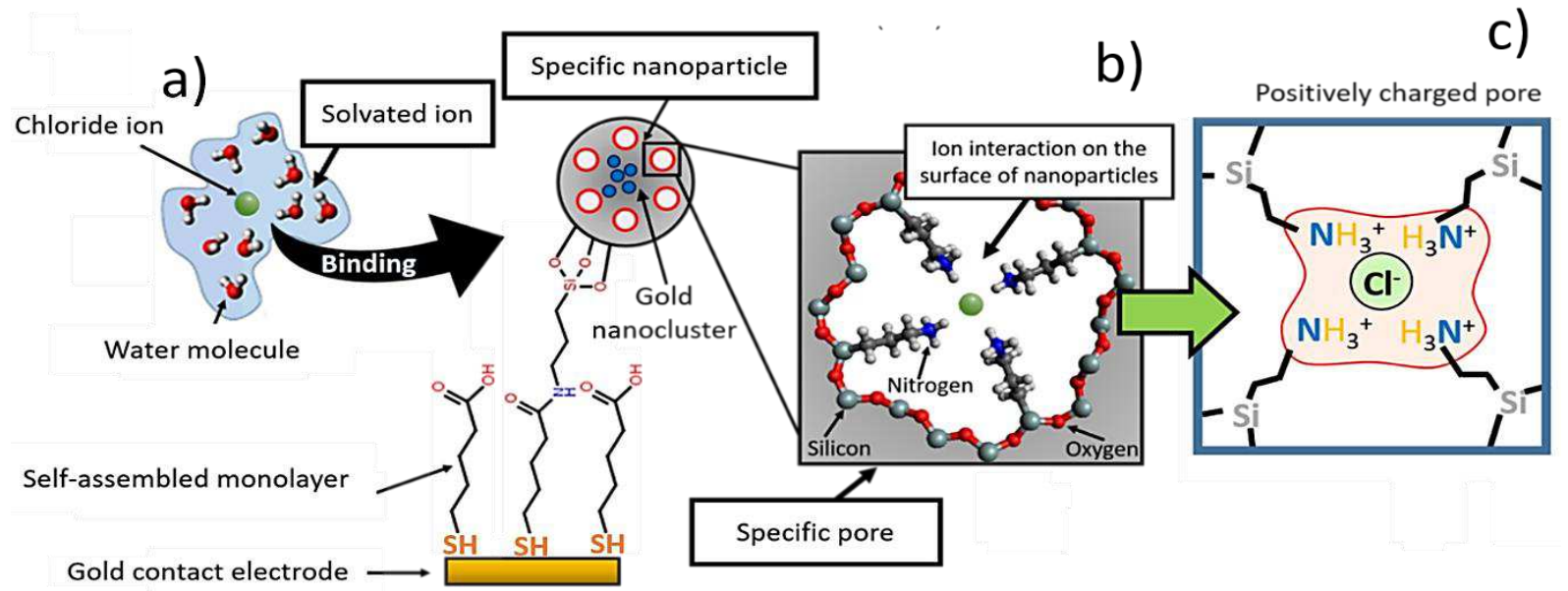

Figure 4. a) Proposed principle of ion recognition process by imprinted nanoparticles. The solvated chloride ion (green) is present in solution with a water shell, and a binding site on the silica surface (red). with a size specific achieved via $\mathrm{HCl}$ templating process to chloride ions and amine groups for the complexation is distributed around the silica-gold nanoparticlulate film surface. b) the ion is complexed in the binding site containing the amine groups (blue), allowing the measurement of its concentration by measuring the changes in the potential. c) Molecular representation of the possible interaction process taking place in the pore cavity of the silica shell between the positively charged amine groups and the chloride ions. The red area indicates the presence of a positively charged environment.

Ion imprinted silica/gold nanoparticles on the sensing layer can address the thickness and sensitivity limitations of conventional membrane-based ion-selective electrodes by offering specific binding sites directly exposed to the sample. Consequently, the sensitivity of the electrodes will be triggered by surface effects, due to the adsorption of chloride ions onto the sensitive silica-gold nanoparticles, instead of the traditional bulk diffusion. This new mechanism allows the sensitivity to go beyond the traditional Nernst limit and permit the size of the sensing electrodes can be reduced. Gold nanoclusters were here employed due to their low charge transfer and electrical resistances and their affinity towards chloride ions ${ }^{[44,45]}$. 
These gold nanoaggregates provided support for the formation of the selective silica shell during the synthesis of the films as depicted in Figure S.7. In addition, such gold nanoaggregates improved the conductivity of the nanoparticles, since the low conductivity of silica could hinder the electrochemical transduction of the sensors. Such effect of improved conductivity has been reported in the case of molecularly imprinted sensors ${ }^{[57]}$. In the present work, the use of gold was fundamental for improving the sensitivity rates of the ion sensors by improving the signal transduction. When no gold was employed during the sensitivity of the devices towards $\mathrm{Cl}^{-}$ions was $-66 \pm 7 \mathrm{mV} \cdot \log \left[\mathrm{Cl}^{-}\right]^{-1}$ when using $\mathrm{NaCl}$ salts for the calibration. This value was significantly lower than the value reported when gold was used as the core material for the ionically imprinted nanoparticles, which was in the range of -186 $\mathrm{mV} \cdot \log \left[\mathrm{Cl}^{-}\right]^{-1}$, confirming the role of the gold nanoaggregates in the sensing.

During this synthesis, the affinity of the gold surfaces towards chloride ions allowed the selective adsorption of such chloride ions onto the surface of the gold nanoaggregates during the synthesis of the core/shell silica/gold nanoparticles. The presence of such chloride ions onto the gold nanoaggregates facilitated the interaction of such anions with the TEOS and APTES monomers during the condensation onto the gold nanoaggregates (Figure S.7). Thus, the presence of gold nanoaggregates during the synthesis of the core/shell silica/gold nanoparticles was necessary for the formation of the selective silica shell.

This novel chloride ion sensing concept with a new sensing architecture demonstrated here for the first time using a chloride ion-specific electrode based on an amine-functionalized ion imprinted porous silica shell with nano pore size (circa. $1.4 \mathrm{~nm}$ in radius) being synthesized onto gold nanoclusters. This structure allowed the miniaturization of the device while ensuring a proper signal transduction and enhanced the selectivity of the device. APTES was used as the recognition agent by quaternisation of the amines present on its molecular 
structure for the sensing $\mathrm{Cl}^{-}$species through the use of $\mathrm{HCl}$, generating a positively charged surface on the imprinted nanoporous silica shell. The use of these positively charged APTES monomers reinforced the electrostatic interactions between the silica/gold nanoparticles and the target chloride anion.

The performance of this ion sensor remained stable at relatively low $\mathrm{pH}$ values (3-7) However, at a higher $\mathrm{pH}$ values, in the range of 11 , the sensitivity of the devices decreased, from $-186 \pm 10 \mathrm{mV} \cdot \log \left[\mathrm{Cl}^{-}\right]^{-1}$ when a $\mathrm{pH}$ of 7 was used up to a standard Nernst sensitivity of $-54 \pm 4 \mathrm{mV} \cdot \log \left[\mathrm{Cl}^{-}\right]^{-1}$ when the $\mathrm{pH}$ reached 11 (Figure S.8). This decrease in the sensitivity was a consequence of the de-quaternisation of the APTES, which takes place at basic $\mathrm{pH}$ values. This effect was reported by Hamza et al. ${ }^{[58]}$, who described the higher absorption rate of uranium ions in the presence of quaternary amine groups. As such, the involvement of the quaternary amine groups from the APTES in the sensing was confirmed, which allowed the potentiometric detection of chloride ions in the electrolytes. This device configuration could overcome the Nernst sensitivity limit.

\section{Conclusions}

We have demonstrated for the first time the application of ionically imprinted nanomaterials in the form of ultra-thin (e.g. $300 \mathrm{~nm}$ thick) nanostructured films with silica/gold core-shell nanoparticles as sensing element for the detection of $\mathrm{Cl}^{-}$. The device showed a superior sensitivity of $-186.4 \mathrm{mV} \cdot \log \left[\mathrm{C}^{-}\right]^{-1}$. This sensitivity surpassed the limit of the current state-ofthe-art chloride ion-selective electrodes, which tend to have a thermodynamical limit of $59 \mathrm{mV} \cdot \log \left[\mathrm{C}^{-}\right]^{-1}$ under Open-Circuit potential measurements and tend to have a relatively thick sensing layer (typically above $100 \mu \mathrm{m}$ ). Therefore, the novel anion sensing device developed here exhibited a 3-fold higher sensitivity, with a detection limit in the range of $10^{-4}$ M. These sensors were proven to offer a stable signal, with a low drift in the range of $1.1 \mathrm{mV}$ 
$\mathrm{h}^{-1}$ and did not require the current lengthy and laborious pre-conditioning process in the commercial anion sensing devices. Such significant enhancement was possible due to the adsorption of the electrolytes to the specific surface interactions with the ion imprinted nanopores on the silica/gold core-shell nanoparticles and the use of positively charged functional groups. This proposed new sensing mechanism based on the adsorption of ions on the silica/gold core-shell nanoparticles has enabled the miniaturization and fabrication of fully functional ultra-thin sensing films with sub-micrometer dimensions, which is not possible using the current commercial plasticized PVC technology which relying on the bulk diffusion of ions inside the sensing films. These anion sensors also showed an unprecedented selectivity towards chloride ions, as demonstrated by calibrating the response of the sensors towards some of the most common interferences found in these sensors, including $\mathrm{HCO}_{3}{ }^{-}, \mathrm{NO}_{3}{ }^{-}$and $\mathrm{SO}_{4}{ }^{2-}$. In addition, they remained stable up to a $\mathrm{pH}$ of 11 . Such breakthrough would open up the opportunity to the fabrication of tailor-made nanostructured thin films for the selective detection of challenging ionic species, especially anions with high specificity and selectivity, which is critical for a wide range of currently important technologies, including environmental remediation, drug development and energy storage among others.

\section{Experimental Section}

\section{Materials}

Tetraethyl orthosilicate (TEOS), tetra chloroauric acid, citric acid, 3-aminopropyl trimethoxysilane (APTES), 1-Ethyl-3-(3-dimethylaminopropyl) carbodiimide, Nhydroxysuccinimide (NHS), Dimethyl formamide, 16-mercaptohexadecanoic acid, sodium chloride, calcium chloride, potassium chloride, sodium sulfate, potassium carbonate, sodium nitrate and hydrochloric acid were purchased from Sigma Aldrich. All the chemicals were used at HPLC grade unless indicated. 


\section{Fabrication of thin film conducting electrode}

A $50 \mathrm{~nm}$ thin gold film was deposited onto a glass substrate by plasma sputtering (Q150RES, Quorum technologies, UK) using standard conditions. Here, a methacrylate mask was first pre-fabricated using a laser cutter (PLS6.75, Universal Laser Systems, Austria). This mask was designed for the deposition of up to 5 electrodes after positioning them onto $7.5 \times 2.5 \mathrm{~cm}$ glass slides and subsequently to be sputtered with gold. Each individual electrode had a radius of $5 \mathrm{~mm}$.

\section{Fabrication of sensing electrode and device}

The fabrication steps and procedures of the sensing materials and device are summarized in Figure 5 and the details are presented below.

\section{Gold NP synthesis}

The ionically imprinted material consisted of a gold nanocluster aggregate integrated with a nanoporous silica shell. These gold nanoparticles were synthesized by the reduction of tetrachloroauric acid (TCA) with citric acid as reported elsewhere ${ }^{[59]}$. Briefly, a $5 \mathrm{~mL}$ solution of $5 \mathrm{mM}$ TCA was mixed in $90 \mathrm{~mL}$ of DI water under constant stirring and heated up to 80 ${ }^{\circ} \mathrm{C}$. Citric acid $0.5 \%$ in $5 \mathrm{~mL}$ was then added and incubated for $6 \mathrm{~h}$ until a red-colored solution was obtained, indicating the success of the synthesis of Au nanoparticles.

\section{Nanoporous core-shell Silica-Au by nanoimprinting}

Nanoporous ion Imprinted silica nanoparticles were synthesized by mixing $4 \mathrm{mM}$ of APTES and $20 \mathrm{mM}$ of TEOS in an aqueous solution containing $10 \mathrm{~mL}$ of 1:1 ethanol:water. A concentration of $1 \mathrm{mM} \mathrm{HCl}$ was used to provide the $\mathrm{Cl}$ - ionic template while providing an acidic environment, favoring the quaternization of aminated species (Figure 5.b). A volume of $5 \mathrm{~mL}$ of the gold nanoparticle dispersion was then mixed with this solution, and the final 
mixture was incubated overnight at $4{ }^{\circ} \mathrm{C}$. The synthesized nanoparticles were washed twice using DI water and dispersed in DMF. A non-templated control sample was additionally synthesized by a similar approach without using $\mathrm{HCl}$.

\section{Sensing device fabrication}

A self-assembled monolayer of thiolated polycarbons was functionalized onto the gold electrodes (fabricated in 3.2.1) by immersing a solution of $1 \mathrm{mM}$ in ethanol overnight (Figure 1.d). APTES was then directionally grafted using standard EDC/NHS chemistry (Figure 1.e). A solution of $15 \mathrm{mM}$ of APTES amine was mixed with the EDC. A volume of $1 \mathrm{~mL}$ of a DMF solution containing NHS using a ratio of 1:2 of EDC:NHS, was then used onto the gold electrode and was incubated for at least 5 mins. The EDC-containing solution was dropped onto the electrodes and they were incubated for at least $4 \mathrm{~h}$. After grafting, the sensor was washed twice by immersing in a solution a mixture of 1:1 ethanol and water to remove any residuals of the EDC and NHS reagents, and the porous core-shell of silica/Au nanoparticulate dispersion was applied to the functionalized electrode in order to induce the nanoparticle grafting and linking of the silica/Au nanoparticulate dispersion.

\section{Structural characterization of the sensing layers}

The morphology of the synthesized silica/Au nanoparticles synthesized by ion imprinting was characterized using Scanning Electron Microscopy (SEM) (EVO LS15, ZEISS, Germany). High resolution transmission electron microscopy (HRTEM) images of the nanoparticles were also taken using a field emission gun transmission electron microscope (FEG-TEM, JEM2100F, JEOL, Japan) operated at the accelerating voltage of $200 \mathrm{kV}$. The high-angle annular dark-field imaging-Scanning transmission electron microscope (HAADF-STEM) image was obtained with an Annular Dark Field (ADF) detector attached to the TEM at a camera length 
of $8 \mathrm{~cm}$. Stylus profilometer (Dektakxt, Bruker, UK) was also used to determine the thickness of the as-fabricated sensing layer in Figure 5.f).

\section{Chemical analysis and composition characterization of the sensing layers}

Chemical analysis was performed using FTIR (L160000A Perkin Elmer, US) and EDS mapping of the elemental composition was carried out on the sensing layers (Figure 5.f). The surface area and pore size distribution of the ion imprinted silica/Au nanoparticles were determined using BET Surface analyzer (Quantachrome, NOVATouch, UK). 


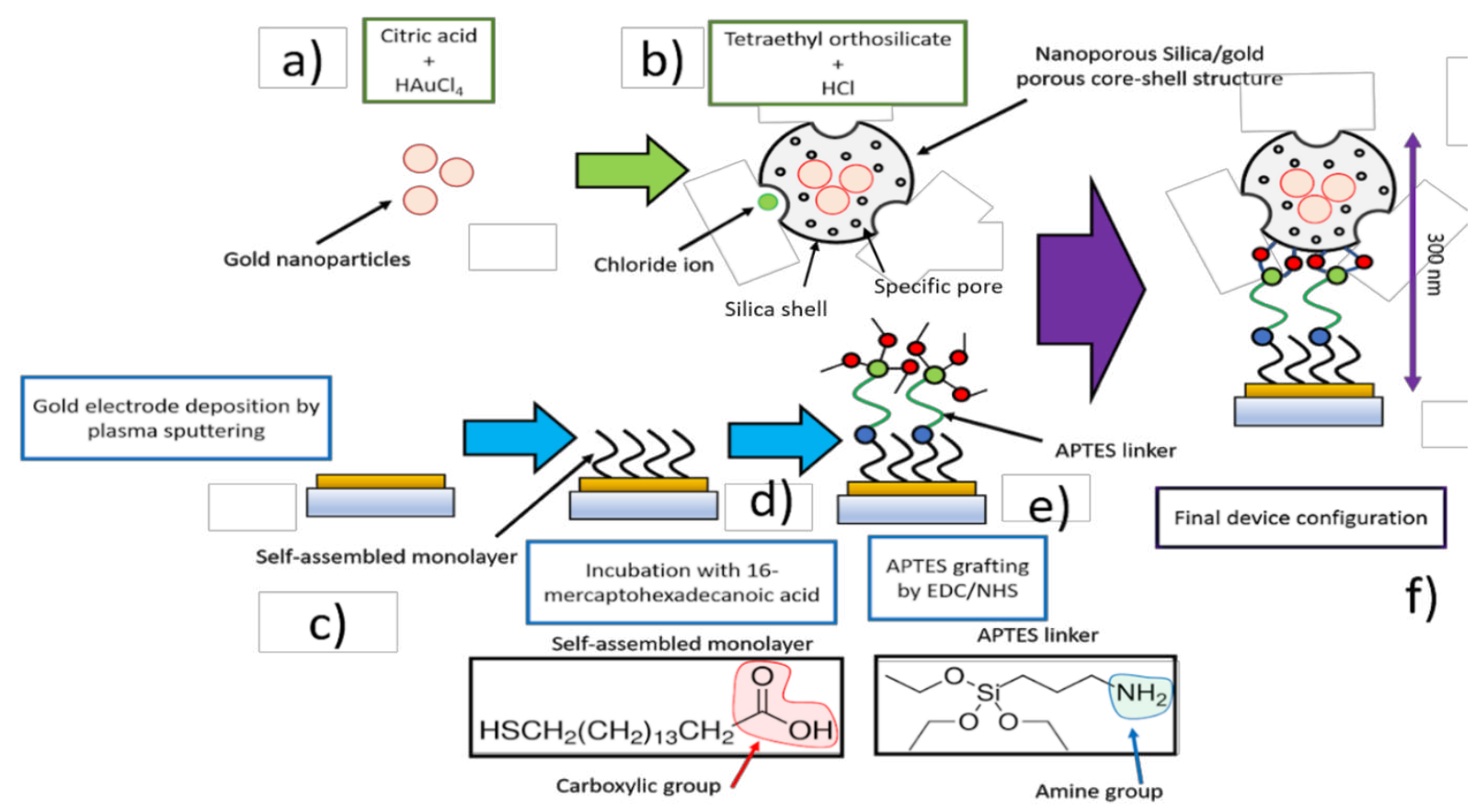

Figure 5. a) Gold NPs synthesis: Au nanoclusters were first synthesized using citric acid as the reducing agent in the presence of $\mathrm{HAuCl}_{4}$. b) Microporous core shell Silica/Au nanoparticles via ionic imprinting method for selective $\mathrm{Cl}^{-}$sensing: The ionically-imprinted imprinted outer silica shell is synthesized using tetraethyl orthosilicate as the structural units and $\mathrm{HCl}$ as the template, using APTES to favor the interaction with the chloride ions. Such APTES is positively charged in the presence of an acid environment as generated by the $\mathrm{HCl}$, increasing the interaction between the porous nanoparticles and the chloride ions. A microporous nanoparticle silica/Au with controlled porosity (circa. $1.5 \mathrm{~nm}$ in pore size) specific towards chloride ions is then developed. c) Electrode fabrication: In parallel, $50 \mathrm{~nm}$ thick gold conducting film was deposited onto glass substrate by plasma sputtering. d) Functionalization of Au electrode: This is achieved by introducing a self-assembled monolayer of 16-mercaptohexadecanoic acid, containing a -SH group that can strongly bind to the gold film, and a carboxyl group(-COOH). e) Linkages: The free -COOH group of the functionalized Au electrode in d) was used to guide the directional attachment of the APTES linker that bridge the electrode with the siloxane groups on the core shell silica-gold synthesized in b) in order to produce f) via the use standard EDC/NHS chemistry; f) A 300 
$\mathrm{nm}$ thick nanostructured sensing film is finally produced specific for the detection of chloride ions.

\section{Sensing performance characterization}

The relative ion intake of the sensing device in Figure 5.f) was determined using a quartz microbalance (Q-sense, Biolin Scientific, Switzerland), and compared to the non-templated control samples (without including $\mathrm{HCl}$ in the precursor solution). The electrochemical analysis and sensing performance was carried out by measuring the Open Circuit Potential (OCP) of the sensors upon being subjected to different solutions with anions $\mathrm{Cl}^{-}, \mathrm{SO}_{4}{ }^{2-}, \mathrm{NO}_{3}{ }^{-}$, $\mathrm{HCO}_{3}{ }^{-}$at different concentrations $\left(10^{-4}, 10^{-3}, 10^{-1}, 1,10,40,80\right.$ and $\left.160 \mathrm{mM}\right)$ and studying the specificity towards interference ions using a standard 3-electrode configuration, with a $\mathrm{Ag} / \mathrm{AgCl}$ reference and a platinum counter electrode. The sensitivity of the electrodes towards $\mathrm{Cl}^{-}$was also repeated by using imprinted nanoparticles with no gold nanoparticles as the core material. The selectivity of the devices was determined by using the matching potentials method. As a verification of the selectivity, the OCP potential of the sensors was recorded while concentrations of $10 \mathrm{mM}$ of the different anions here employed were added. For the determination of the effects of $\mathrm{pH}$ on the sensing performance, $\mathrm{NaOH}$ and $\mathrm{H}_{2} \mathrm{SO}_{4}$ were added to DI water to change the $\mathrm{pH}$ values. 5 different $\mathrm{pH}$ were tested $(3,5,7,9$ and 11$)$, and the sensitivity of the devices using $\mathrm{NaCl}$ solutions at the concentrations above mentioned was determined.

\section{References}

1. Desai, A.S., Karl Swedberg, John J.V.McMurray, Christopher B. Granger, Salim Yusuf, James B. Young, Mark E. Dunlap, Scott D. Solomon, James W. Hainer, Bertil Olofsson, Eric 
L. Michelson, Marc A. Pfeffer. Journal of the American College of Cardiology, 2007. 50(20): p. 1959-1966.

2. Sica, D.A. Deterrent to the Use of Aldosterone Receptor Antagonism or Not, 2009. 53(5): p. 749-750.

3. Wei Gao, Sam Emaminejad, Hnin Yin Yin Nyein, Samyuktha Challa, Kevin Chen, Austin Peck, Hossain M. Fahad, Hiroki Ota, Hiroshi Shiraki, Daisuke Kiriya, Der-Hsien Lien, George A. Brooks, Ronald W. Davis, Ali Javey. Nature, 2016. 529(7587): p. 509-514.

4. Lin, W.-C., Z. Li, and M.A. Burns. Analytical Chemistry, 2017. 89(17): p. 8748-8756.

5. Chen, F., Z.Y. Leong, and H.Y. Yang. Energy Storage Materials, 2017. 7: p. 189-194.

6. Yarong Song, Guangming Jiang, Ying Chen, Peng Zhao, Yimei Tian. Scientific Reports, 2017. 7(1): p. 6865.

7. Adamchuk V.I., Hummel J. W., Morgan M.T., Upadhyaya S.K. Computers and Electronics in Agriculture, 2004. 44(1): p. 71-91.

8. Luke, R.G., Serum Chloride and Bicarbonate Levels in Chronic Renal Failure. Archives of Internal Medicine, 1979. 139(10): p. 1091-1092.

9. Neligan, P.J. and R. O'Donoghue, 56 - How Should Acid-Base Disorders Be Diagnosed and Managed?, in Evidence-Based Practice of Critical Care, C.S. Deutschman and P.J. Neligan, Editors. 2010, W.B. Saunders: Philadelphia. p. 389-396.

10. Winkler, S., et al., Application of ion-sensitive sensors in water quality monitoring. Water science and technology : a journal of the International Association on Water Pollution Research, 2004. 50: p. 105-14.

11. Heller, L. and M. Ben-Yair, Effect of chloride solutions on portland cement. Journal of Applied Chemistry, 1966. 16(8): p. 223-226.

12. Gemene, K.L., M.E. Meyerhoff. Electroanalysis, 2012. 24(3): p. 643-648.

13. Lewenstam, A.. Electroanalysis, 2014. 26(6): p. 1171-1181. 
14. Ryoichi Ishimatsu, Anahita Izadyar, Benjamin Kabagambe, Yushin Kim, Jiyeon Kim, and Shigeru Amemiya. Journal of the American Chemical Society, 2011. 133(40): p. 1630016308.

15. Zdeňka Jarolímová, Tingting Han, Ulriika Mattinen, Johan Bobacka, Eric Bakker. Analytical Chemistry, 2018. 90(14): p. 8700-8707.

16. Zdrachek, E., E. Bakker. Analytical Chemistry, 2019. 91(1): p. 2-26.

17. Schaller, U., E. Bakker, E. Pretsch. Analytical Chemistry, 1995. 67(18): p. 3123-3132.

18. Bakker, E. TrAC Trends in Analytical Chemistry, 2014. 53: p. 98-105.

19. Zsófia Szigeti, Tamás Vigassy, Eric Bakker, Ernö Pretsch. Electroanalysis, 2006. 18(1314): p. 1254-1265.

20. F. Keplinger, R. Glatz, A. Jachimowicz, G. Urban, F. Kohl, F. Olcaytug, O. J. Prohaska. Sensors and Actuators B: Chemical, 1990. 1(1): p. 272-274.

21. Sanggil, H., Yamamoto, S., Polyravas, A. Malliaras, G.. Advanced Materials. 2020. p. 2004790

22. J. M. Rothberg, W. Hinz, T. M. Rearick, J. Schultz, W. Mileski, M. Davey, J. H. Leamon, K. Johnson, M. J. Milgrew, M. Edwards, J. Hoon, J. F. Simons, D. Marran, J. W. Myers, J. F. Davidson, A. Branting, J. R. Nobile, B. P. Puc, D. Light, T. A. Clark, M. Huber, J. T. Branciforte, I. B. Stoner, S. E. Cawley, M. Lyons, Y. Fu, N. Homer, M. Sedova, X. Miao, B. Reed, J. Sabina, E. Feierstein, M. Schorn, M. Alanjary, E. Dimalanta, D. Dressman, R. Kasinskas, T. Sokolsky, J. A. Fidanza, E. Namsaraev, K. J. McKernan, A. Williams, G. T. Roth, J. Bustillo, Nature 2011, 475, 348.

23. J. V. Raimondo, R. J. Burman, A. A. Katz, C. J. Akerman, Front. Cell. Neurosci. 2015, 9, 419.

24. Shklovskii, B.I. Physical Review E, 1999. 60(5): p. 5802-5811.

25. Grosberg, A.Y., T.T. Nguyen, B.I. Shklovskii. Reviews of Modern Physics, 2002. 74(2): p. $329-345$. 
26. R. Sivakumarasamy, R. Hartkamp, B. Siboulet, J.-F. Dufrêche, K. Nishiguchi, A. Fujiwara, N. Clément. Nature Materials, 2018. 17(5): p. 464-470.

27. Abdelwahab, O., N.K. Amin, E.S.Z. El-Ashtoukhy. Chemical Engineering Research and Design, 2013. 91(1): p. 165-173.

28. Risto Koivula, Jukka Lehto, Leena Pajo, Thomas Gale, Heikki, Leinonen. Hydrometallurgy, 2000. 56(1): p. 93-108.

29. B. L. Rivas, H. A. Maturana, S. Villegas, E. Pereira. Polymer Bulletin, 1998. 40(6): p. 721-728.

30. Dudzinska, M.R., D.A. Clifford. Reactive Polymers, 1991. 16(1): p. 71-80.

31. Junqing $\mathrm{Fu}$, Lingxin Chen, Jinhua Lia, Zhong Zhangac. Journal of Materials Chemistry A, 2015. 3(26): p. 13598-13627.

32. Branger, C., W. Meouche, A. Margaillan. Reactive and Functional Polymers, 2013. 73(6): p. 859-875.

33. Dakova, I., et al., Solid phase selective separation and preconcentration of $\mathrm{Cu}$ (II) by Cu(II)-imprinted polymethacrylic microbeads. Analytica Chimica Acta, 2007. 584(1): p. 196203.

34. Y1lmaz, V., O. Hazer, Ş. Kartal. Talanta, 2013. 116: p. 322-329.

35. Dam, H.A., D. Kim. Industrial \& Engineering Chemistry Research, 2009. 48(12): p. $5679-5685$.

36. Zhang, N., B. Hu. Analytica Chimica Acta, 2012. 723: p. 54-60.

37. Mohammad Behbahani, Mozhgan Barati, Majid Kalate Bojdi, Ali Reza Pourali, Akbar Bagheri, Nasim Akbari Ghareh Tapeh. Microchimica Acta, 2013. 180(11): p. 1117-1125.

38. Wang, Z., G. Wu, C. He. Microchimica Acta, 2009. 165(1): p. 151-157.

39. Xiangbing Zhu, Yuemei Cui, Xijun Chang, Xiaojun Zou, Zhenhua Li. Microchimica Acta, 2009. 164(1): p. 125-132.

40. Jenkins, A.L., O.M. Uy, G.M. Murray. Analytical Chemistry, 1999. 71(2): p. 373-378. 
41. Kala, R., T. Prasada Rao. Journal of Separation Science, 2006. 29(9): p. 1281-1287.

42. Bomar, E.M., G.S. Owens, G.M. Murray. Chemosensors, 2017. 5(1): p. 2.

43. Alizadeh, T., K. Atayi. Materials Chemistry and Physics, 2018. 209: p. 180-187.

44. Laurinavichyute, V.K., S. Nizamov, V.M. Mirsky. Chem. Phys. Chem, 2017. 18(12): p. $1552-1560$

45. Bradbury, C.R., J. Zhao, D.J. Fermín. The Journal of Physical Chemistry C, 2008. 112(27): p. 10153-10160.

46. Chengcheng Kang, Weiming Li, Lei Tan, He Li, Chaohai Weic, Youwen Tang. Journal of Materials Chemistry A, 2013. 1(24): p. 7147-7153.

47. Zulei Zhang, Xingdi Zhang, Dechao Niu, Yongsheng Li, Jianlin Shi. Journal of Hazardous Materials, 2017. 328: p. 160-169.

48. Sen Yang, Jun Qian, Liangju Kuang, Daoben Hua. ACS Applied Materials \& Interfaces, 2017. 9(34): p. 29337-29344.

49. Tripta Kamra, Shilpi Chaudhary, Changgang Xu, Lars Montelius, Joachim Schnadt, Lei Ye. Journal of Colloid and Interface Science, 2016. 461: p. 1-8.

50. Dezhi Zhang, Hisham E. Hegab, Yuri Lvov, L. Dale Snow, James Palmer. SpringerPlus, 2016. 5(1): p. 48-48.

51. Marcin Guzinski, Jennifer M. Jarvis, Bradford D. Pendley, Ernő Lindner. Analytical Chemistry, 2015. 87(13): p. 6654-6659.

52. Chunling Wang, Hongyan Yuan, Zhijuan Duan, Dan Xiao. Scientific Reports, 2017. 7(1): p. 44771.

53. M Brunetti 1, L Terracina, M Timio, P Saronio, E Capodicasa . Journal of nephrology, 2001. 14: p. 27-31.

54. Gary D Miller, Anthony P Marsh, Robin W Dove, Daniel Beavers, Tennille Presley, Christine Helms, Erika Bechtold, S Bruce King, Daniel Kim-Shapiro. Nutrition Research, 2012. 32(3): p. 160-168. 
55. Basile, C., L. Rossi, C. Lomonte. Kidney International, 2016. 89(5): p. 1008-1015.

56. Y. Su, S. Xia, R. Wang, L. Xiao. Hormone Metabolism and Signaling in Plants ,2017. 431470.

57. Li, Y., Liu, Y., Liu, J., Liu, J., Tang, H., Cao, C., Zhao, D., Ding, Y. Scientific Reports. 2014.

58. Hamza, M. Journal of Radioanalytical and Nuclear Chemistry, 2019. 1-14.

59. Dar, A.I., S. Walia, A. Acharya. Journal of Nanoparticle Research, 2016. 18(8): p. 233

\section{Supporting Information}




\section{A miniaturised sensing device with ionically imprinted nanostructured films for the ultrasensitive detection of ions}

Antonio Ruiz-Gonzalez ${ }^{a}$, Cao Xun ${ }^{b}$, Huang Yizhong ${ }^{b}$, and Kwang Leong Choy ${ }^{a, *}$

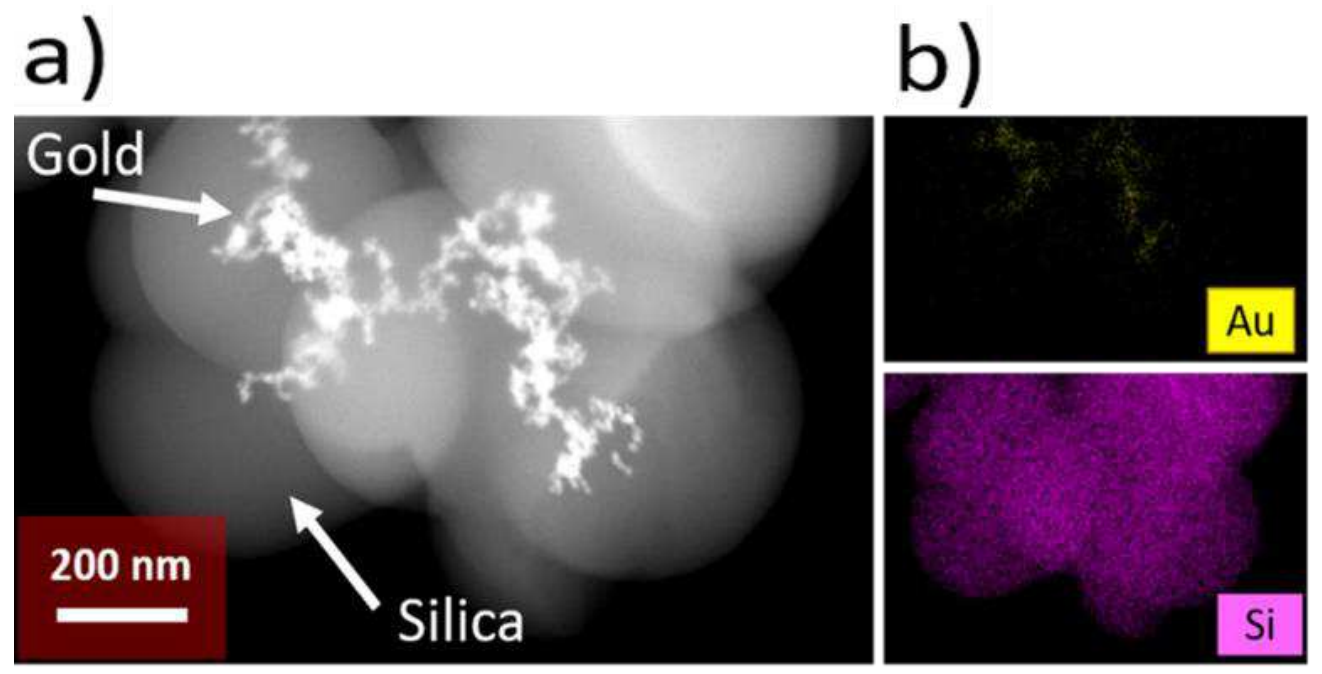

Figure S.1. a) HAADF-STEM revealing the distribution of silicon and gold atoms within the ionically imprinted gold/silica nanoparticles using a $\mathrm{Z}$ contrast. b) EDX mapping of Si, $\mathrm{O}$ and $\mathrm{Au}$ elements contained within the silica nanostructure. 


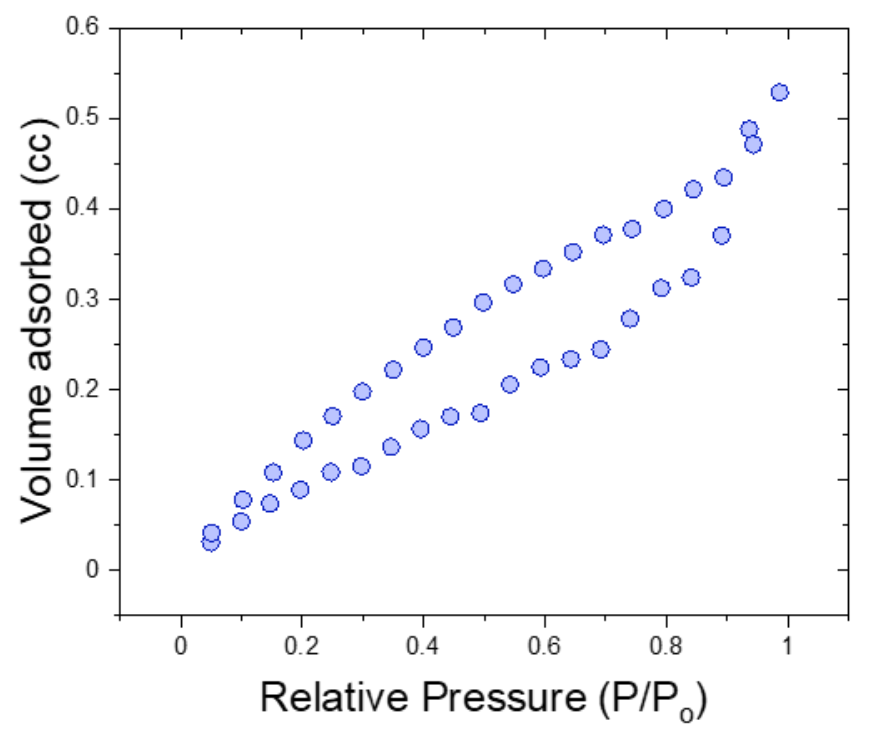

Figure S.2. Isotherms for the ionically imprinted gold/silica nanoparticles obtained during the BET process. Such isotherms were used to calculate the average surface area of the nanoparticles by using the BET equations, which correlates the relative pressure with the number of adsorbed gas molecules, which is related with the surface area of the material. In this case, the surface area of the present material was $108 \mathrm{~m}^{2} \mathrm{~g}^{-1[1]}$. 


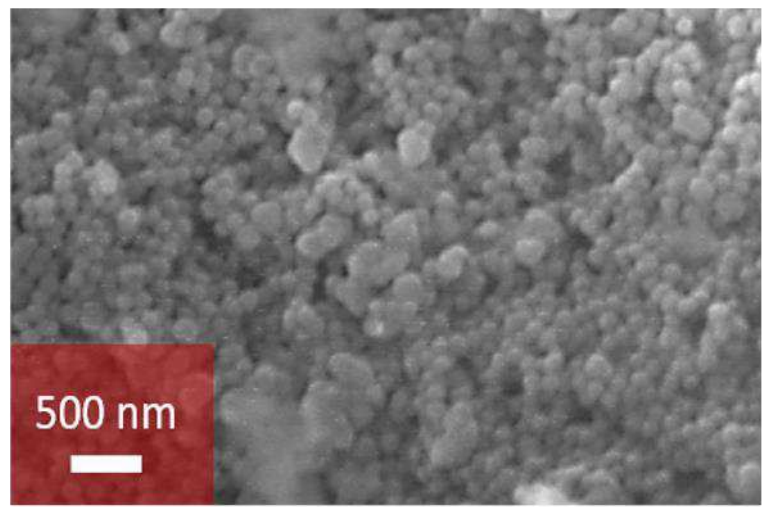

a)

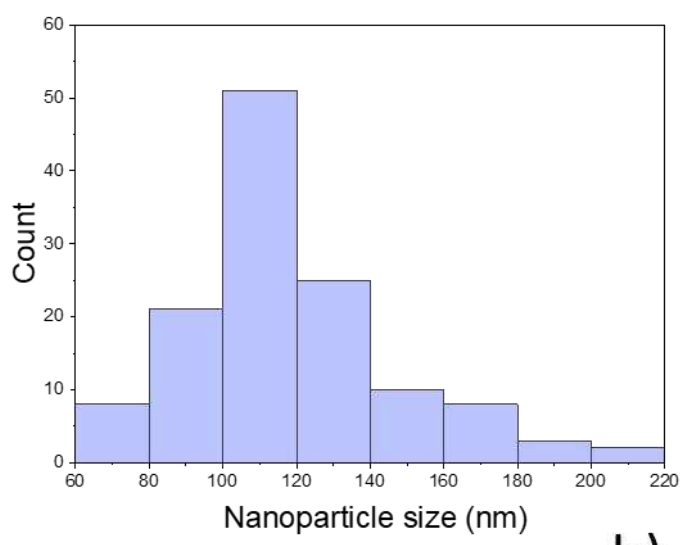

b)

Figure S.3. a) SEM visualization of the ionically imprinted gold/silica nanoparticles employed for the measurement of chloride ions. b) The SEM micrograph in a) was employed for the determination of the nanoparticle size by measurement of these ionically imprinted gold/silica nanoparticles using ImageJ. $40 \%$ of the -particles have a typical size in the range of $100-120 \mathrm{~nm}$ and the mean size was $118 \pm 29 \mathrm{~nm}$. 


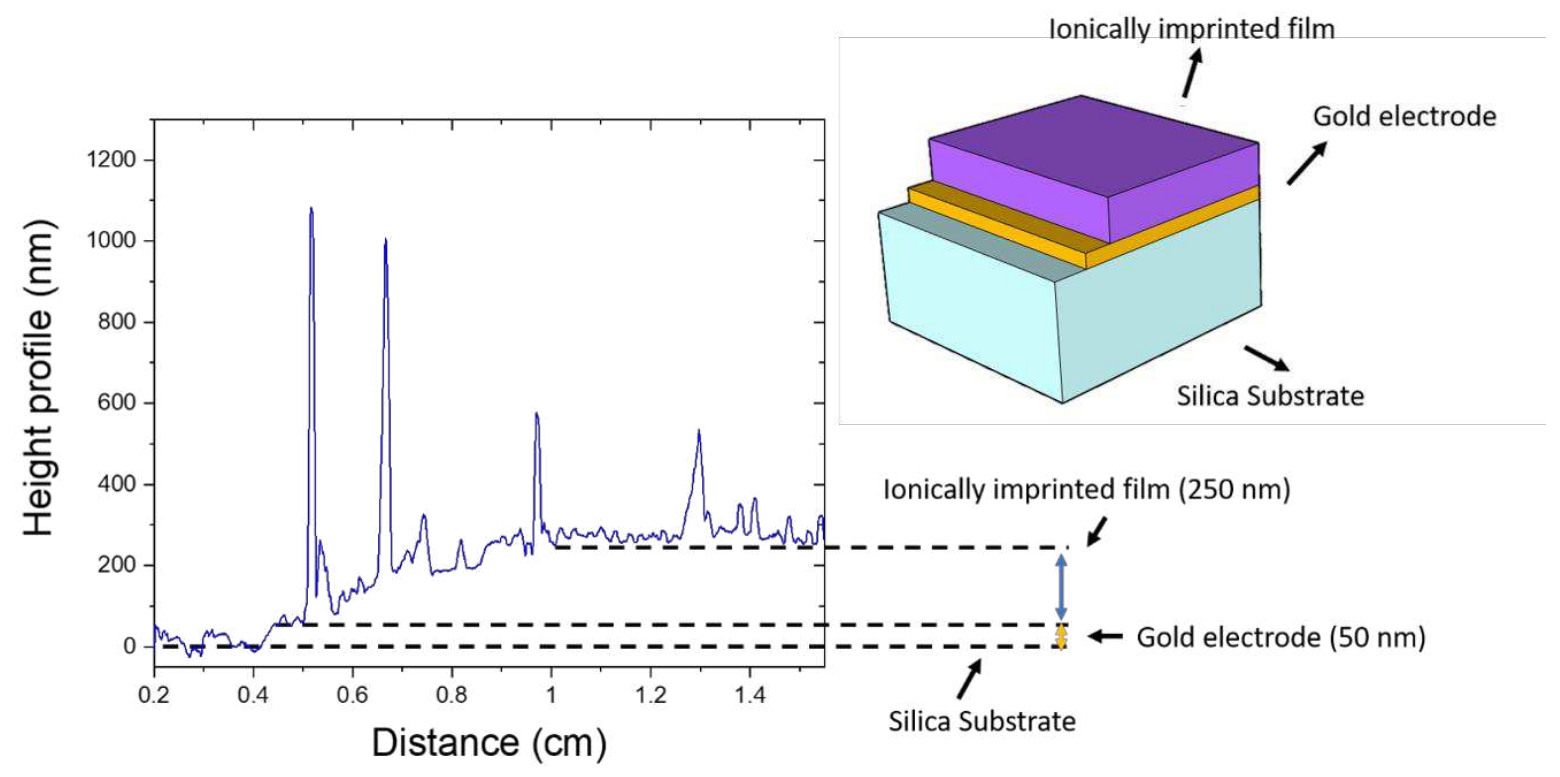

Figure S.4. Profile of the full device containing the gold/silica core/shell imprinted nanoparticles grafted onto a $50 \mathrm{~nm}$ thick gold electrode on a silica substrate as measured by a stylus profilometer. In the first part of the profile (up to $0.4 \mathrm{~cm}$ ) the stylus is placed on the silica substrate, and the height profile is closed to $0 \mathrm{~nm}$. The thickness of the full device containing the gold/silica core/shell imprinted nanoparticles is the measured, with a size closed to $300 \mathrm{~nm}$, including the $50 \mathrm{~nm}$ thick gold electrode deposited by plasma sputtering. The inset shows a schematic diagram of this sensing film, illustrating the structure of the full device. 


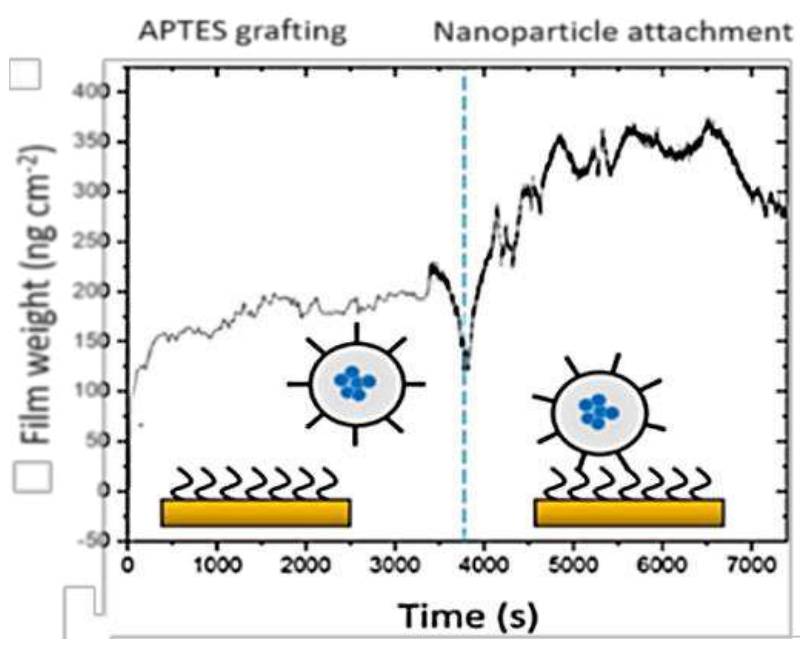

Figure S.5. Quartz microbalance monitoring of the weight changes of the gold electrodes during the functionalisation process with the self-assembled film and the silica/gold nanoparticles. Initially, the gold electrode was functionalised with a self-assembled monolayer containing 16-mercaptohexadecanoic acid after the exposure of the films to the nanoparticles, an increase in the weight of $294 \pm 3 \mathrm{ng} \mathrm{cm}^{-2}$ was observed. 
Table S.1. Table comparison between the selectivity coefficients achieved in the present work and the reported values in the literature calculated by the matched potentials method ${ }^{[2]}$. Here, smaller values represent a higher selectivity.

\begin{tabular}{|c|c|c|c|}
\hline \multirow{2}{*}{ Anion } & $\begin{array}{c}\text { Present work } \\
\log \left(K_{C l, j}^{p o t}\right)\end{array}$ & $\begin{array}{c}\text { Reported results } \\
\log \left(K_{C l, j}^{p o t}\right)\end{array}$ & Ref \\
\hline $\mathrm{SO}_{4}{ }^{2-}$ & -2.4 & -2 & {$[2]$} \\
\hline $\mathrm{NO}_{3}{ }^{-}$ & -2.1 & +0.2 & {$[2]$} \\
\hline $\mathrm{HCO}_{3}{ }^{-}$ & -2.6 & -2.6 & {$[2]$} \\
\hline $\mathrm{Br}^{-}$ & -2.0 & +0.2 & {$[2]$} \\
\hline $\mathrm{F}^{-}$ & -2.1 & - & {$[2]$} \\
\hline $\mathrm{OH}^{-}$ & -6.4 & - & {$[2]$} \\
\hline
\end{tabular}




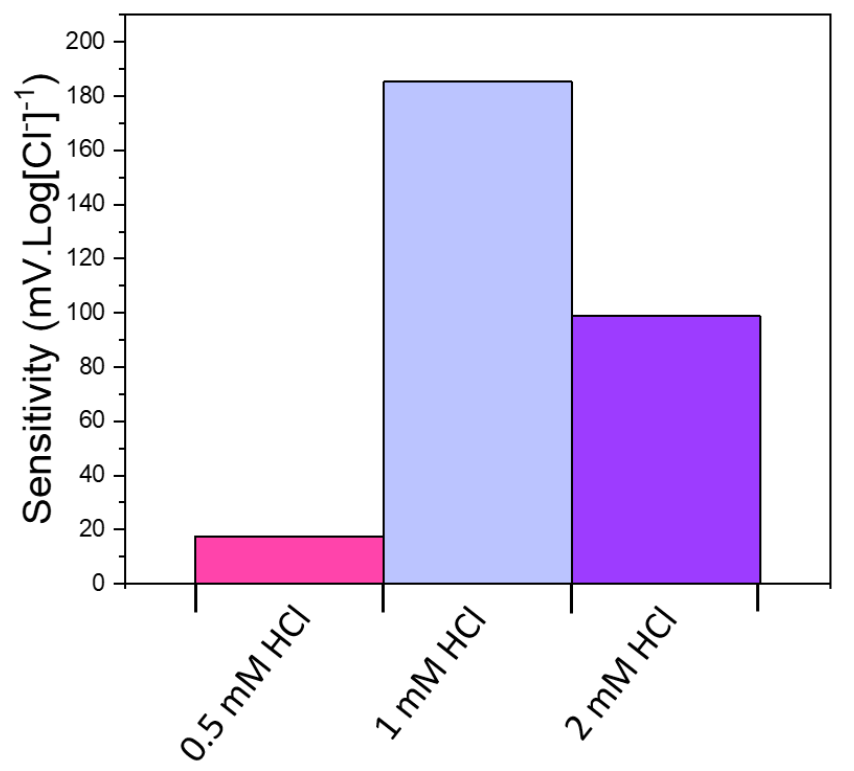

Figure S.6. Comparison of the profile of sensitivities achieved for different Ionically imprinted gold/silica nanoparticles synthetized using different concentrations of $\mathrm{HCl}$ as the template ion. Lower sensitivities were achieved in the case of 0.5 and $2 \mathrm{mM}$ with sensitivities-17.4 and $-99.1 \mathrm{mV} \cdot \log \left[\mathrm{Cl}^{-}\right]^{-1}$. respectively. On the contrary, an optimal composition was obtained using $1 \mathrm{mM} \mathrm{HCl}$, achieving a maximum sensitivity of $-186.7 \mathrm{mV} \cdot \log \left[\mathrm{Cl}^{-}\right]^{-1}$. 


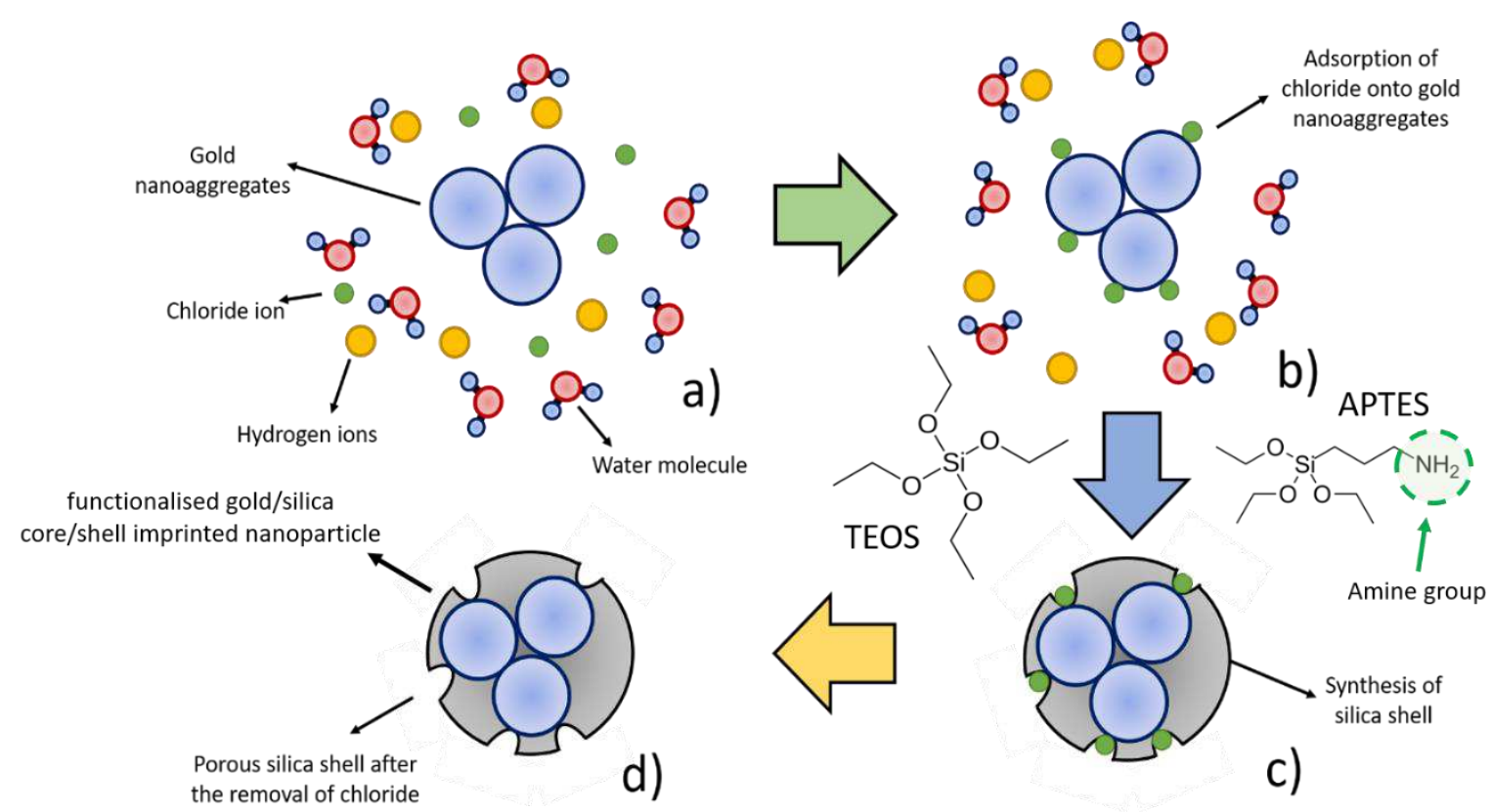

Figure S.7. Schematic representation of the role of gold nanoaggregates during the synthesis of the gold/silica core/shell imprinted nanoparticles. a) Initially, the gold nanoaggregates are dispersed in an aqueous solution, and the template chloride are introduced. b) Due to the affinity of such gold nanoaggregates towards the chloride ions, such ions selectively adsorb onto the surface of the gold nanoaggregates. Such adsorption will prevent the formation of nanoimprinted silica nanoparticles with selectivity towards other components present in the solution (e.g. hydrogen ions, water) due to the core-shell structure here synthetized. c) After adding the TEOS and APTES monomers to the solution, a shell containing the functionalised silica monomers with the amine groups provided by the APTES. This shell is porous, with a radius circa. $1.4 \mathrm{~nm}$ and contains the chloride ions initially, since they have been used as a template. d) The chloride ions contained within the nanopores formed by the functionalised silica shell are removed by washing, leaving a functionalised gold/silica core/shell imprinted nanoparticle that can be used for the sensing of chloride ions. 


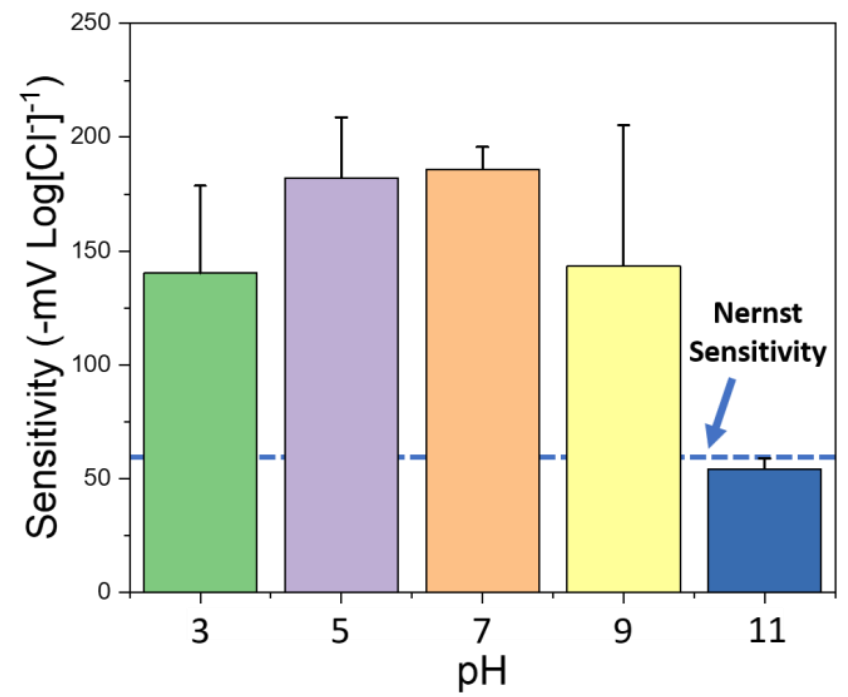

Figure S.8. Sensitivity towards chloride ions of the ion sensing devices containing the gold/silica ionically imprinted nanomaterials when subjected to different $\mathrm{pH}$ values. Such $\mathrm{pH}$ was tailored using $\mathrm{NaOH}$ and $\mathrm{H}_{2} \mathrm{SO}_{4}$. The Nernst sensitivity limit has been indicated. 


\section{Reference}

1. Lowell, S., et al., Surface Area Analysis from the Langmuir and BET Theories, in Characterization of Porous Solids and Powders: Surface Area, Pore Size and Density, S. Lowell, et al., Editors. 2004, Springer Netherlands: Dordrecht. p. 58-81.

2. Xiao, K.P., et al., A Chloride Ion-Selective Solvent Polymeric Membrane Electrode Based on a Hydrogen Bond Forming Ionophore. Analytical Chemistry, 1997. 69(6): p. 1038-1044. 


\section{Figures}

a)

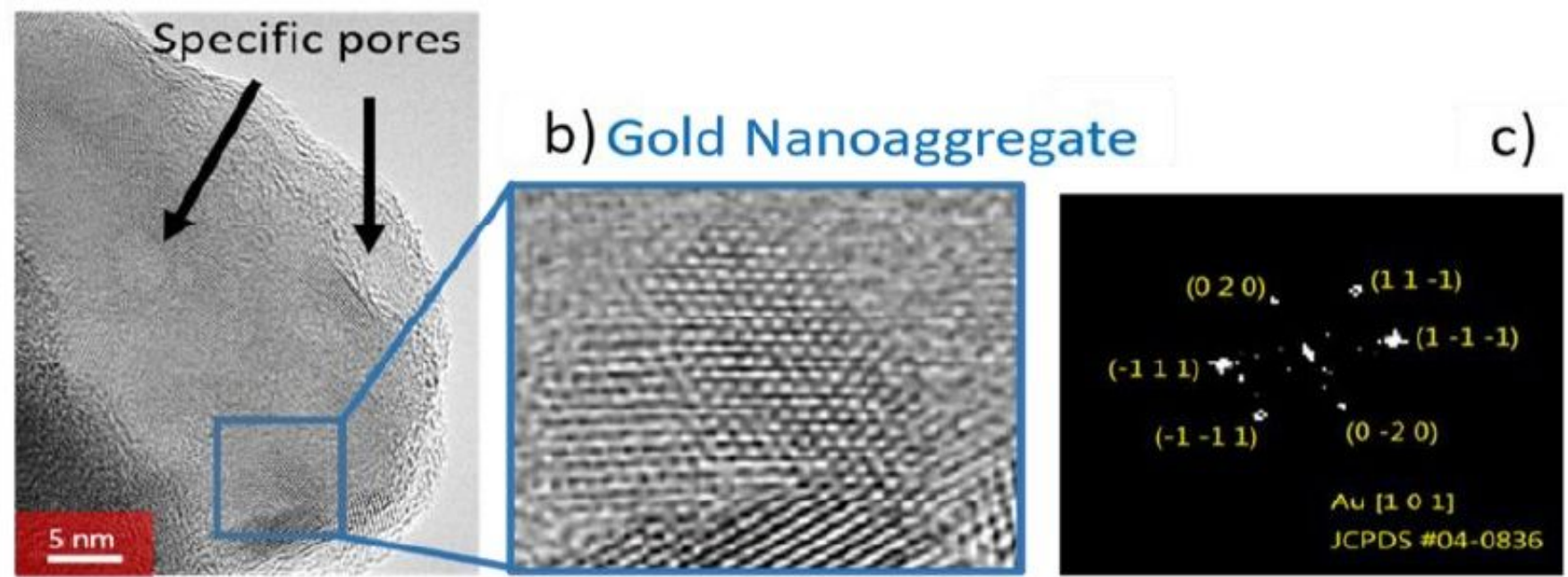

d)

e)
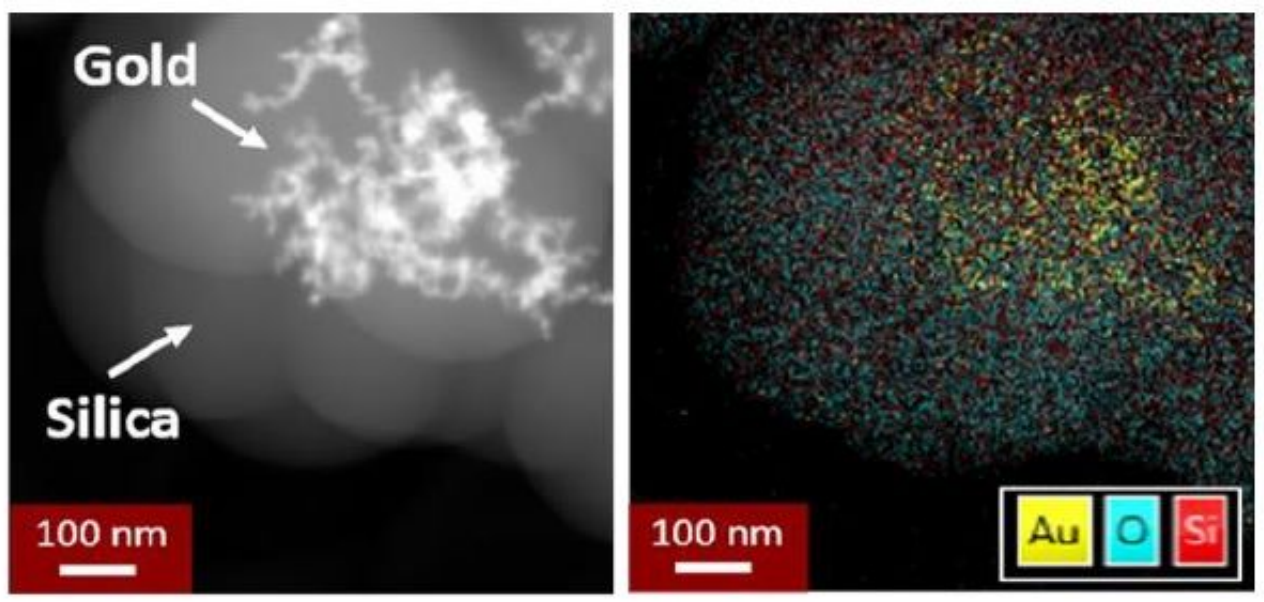

Figure 1

HRTEM visualization of the silica-Au core-shell structure. a) Detail of the surface of the ionically imprinted silica onto the gold nanoclusters where the pore structure is highlighted with pore diameter typically around $3 \mathrm{~nm}$. b) A gold nanoaggregate is magnified, allowing the visualization of the crystalline structure.

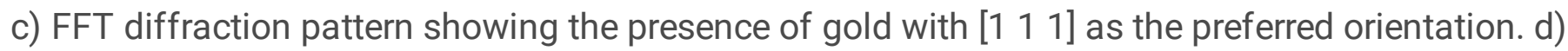
HAADF-STEM revealing the distribution of both silicon and gold elements across the core-shell structure using a Z contrast. e) EDX mapping of Au (yellow) and Si (Pink) indicating the distribution of both elements within the ionically imprinted Gold/silica nanoparticles. 

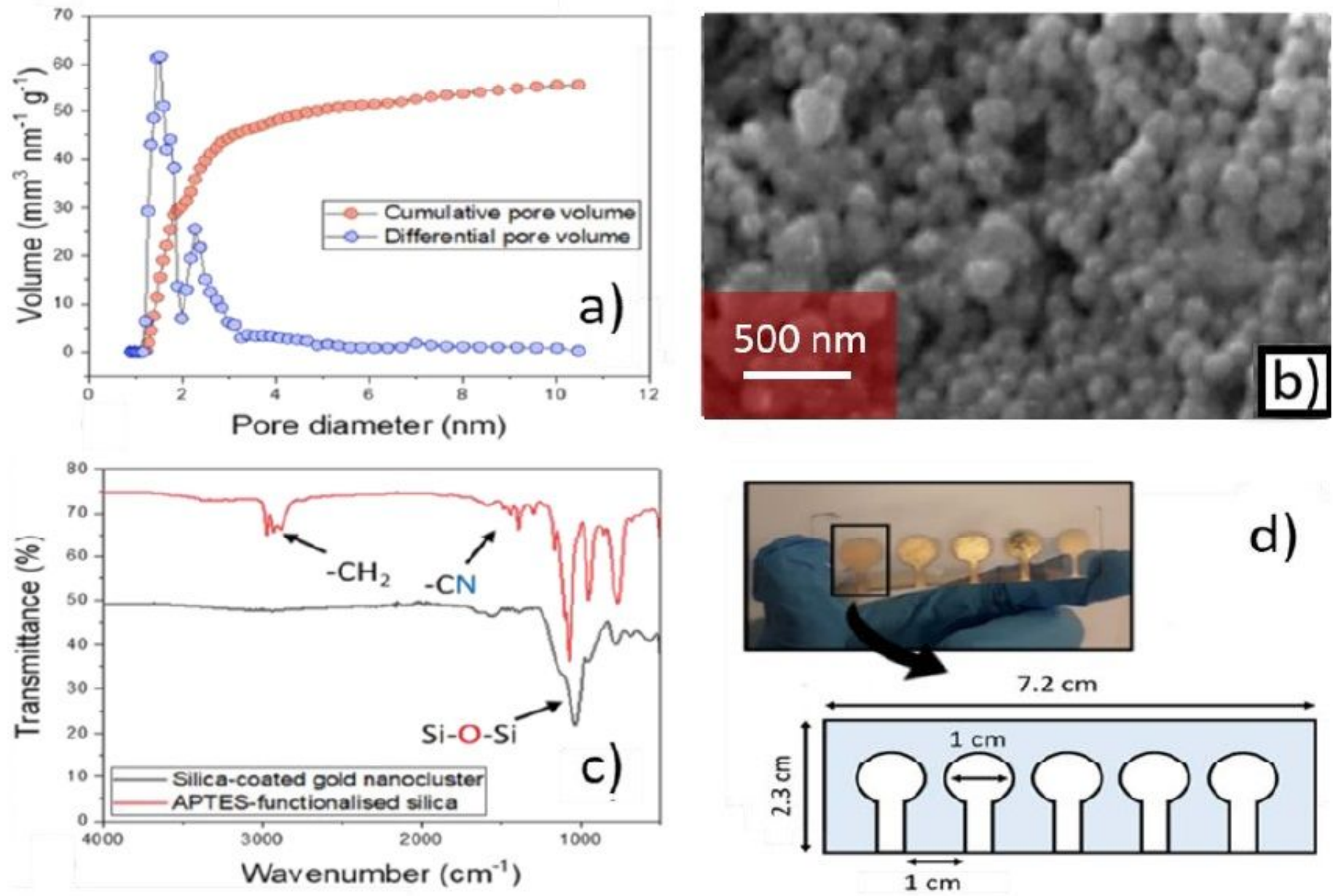

Figure 2

a) Cumulative and differential pore volume obtained by BET, showing a higher presence of pores in the $2.8 \mathrm{~nm}$ range. b) SEM image of the silica encapsulated gold nanoparticles. c) FTIR spectrum of the silicaencapsulated gold when compared with the one obtained for APTES-functionalized silica. The representative peaks of the Si-O-Si bonds present in silicon dioxide (1032 and $778 \mathrm{~cm}-1)$ and APTES ($\mathrm{CH} 2$ at $3000 \mathrm{~cm}-1$ and $-\mathrm{CN}$ in the range of $1600 \mathrm{~cm}-1$ ) are highlighted. d) Picture of the thin film sensing device containing the grafted ionically imprinted silica nanoparticles on the surface of gold electrodes. The dimensions of the sensors are highlighted. 

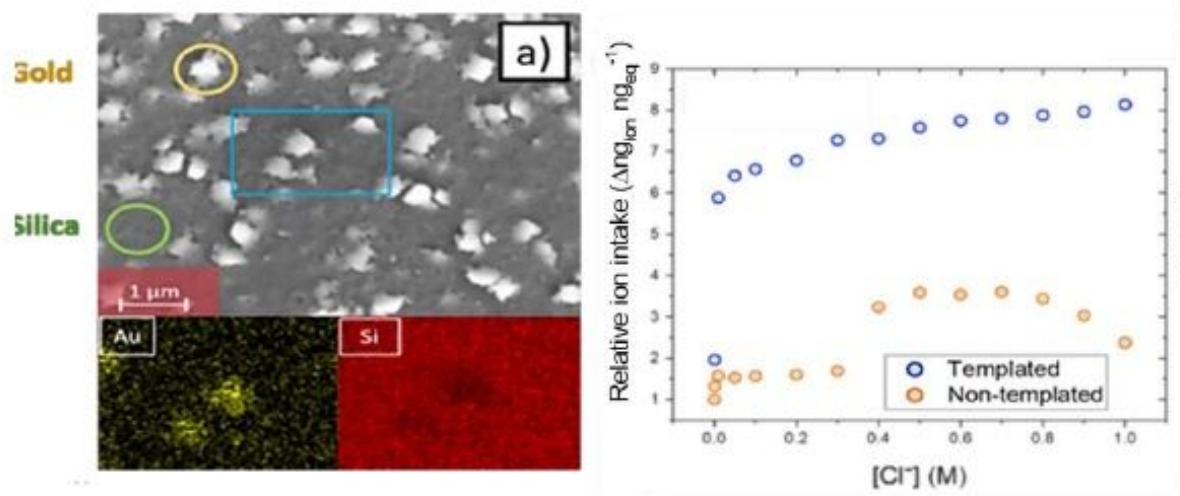

b)

c)
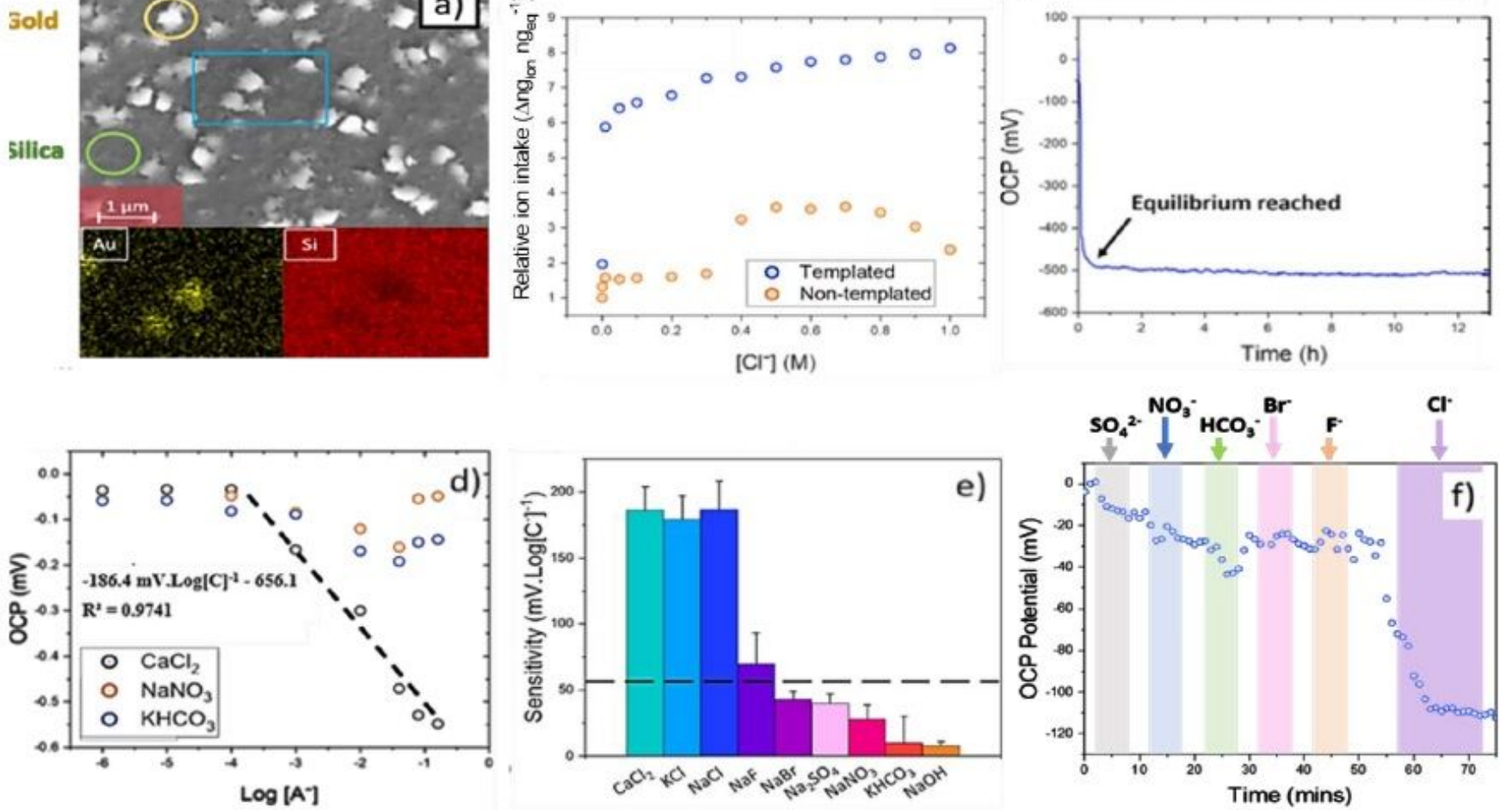

\section{Figure 3}

a) SEM image of the sensing film containing the ion imprinted silica/gold nanoparticulate surface morphology. The EDS elemental mapping of gold and silica in the highlighted area is also shown. b) Relative absorption of chloride ions by the ion-imprinted electrodes grafted onto gold surfaces as measured by quartz microbalance. These values were measured by subjecting the film to different concentrations of $\mathrm{NaCl}$, and the results were normalized by the first observed weight value, when only water was used. c) OCP monitoring of the chloride-selective sensors over $13 \mathrm{~h}$ using $\mathrm{KCl}$. The equilibrium point of the sensor in $\mathrm{KCl}$ is indicated. d) Recorded electrochemical potentials at different concentrations of different anions. The calculation of the sensitivity of the devices was exemplified by taking the linear range of the calibration plot. e) Sensitivity profiles of the ionic imprinting-based electrodes after testing with different metal chlorides $(\mathrm{NaCl}, \mathrm{KCl}, \mathrm{CaCl} 2)$ and interference anions from $\mathrm{Na} 2 \mathrm{SO} 3, \mathrm{NaNO} 3$ and KHCO3. f) Monitoring of the OCP signal of a sensing device containing the gold/silica ionically imprinted nanoparticles when subjected to a mixed concentration of anions. The result of cumulatively adding 10 mM S042- (grey), 10 mM of NO3- (blue), 10 mM HCO3- (green), $10 \mathrm{mM} \mathrm{Br-(pink)} \mathrm{and} 10 \mathrm{mM} \mathrm{F-} \mathrm{(orange)}$ was measured. Only a significant change in the potentiometric signal can be observed when $\mathrm{Cl}$-is added. 


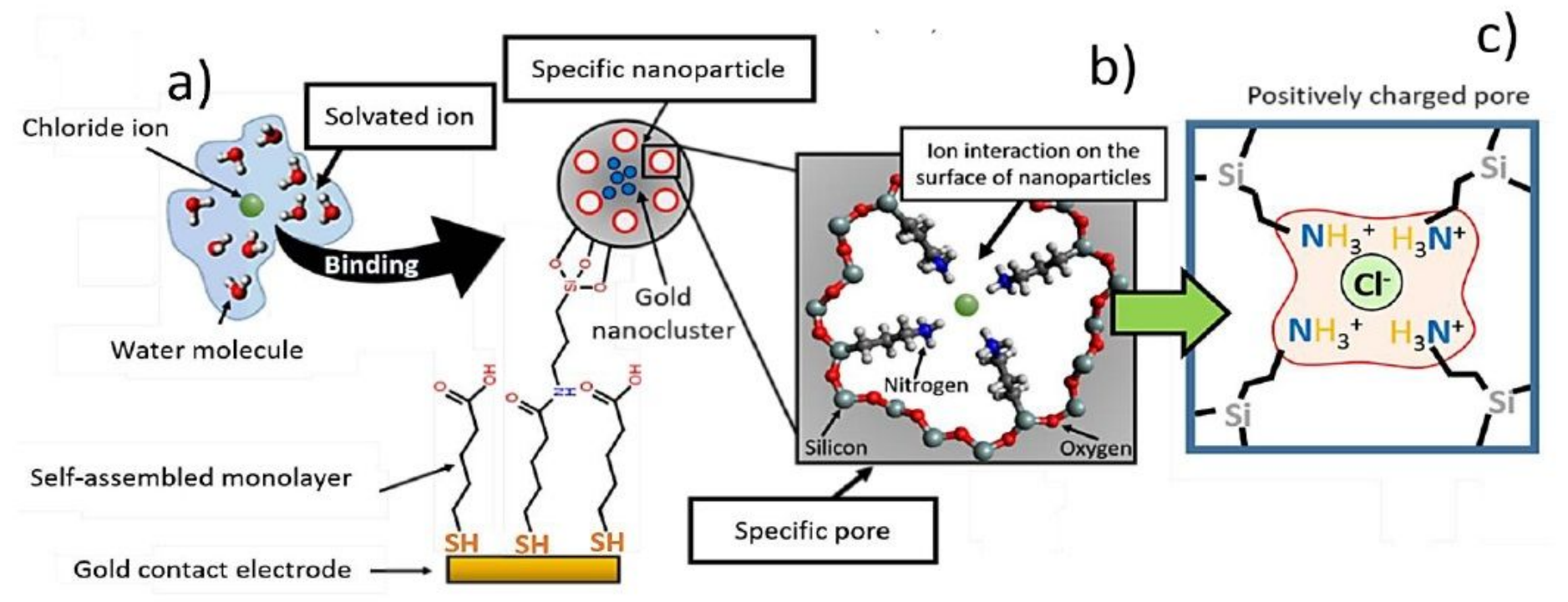

Figure 4

a) Proposed principle of ion recognition process by imprinted nanoparticles. The solvated chloride ion (green) is present in solution with a water shell, and a binding site on the silica surface (red). with a size specific achieved via $\mathrm{HCl}$ templating process to chloride ions and amine groups for the complexation is distributed around the silica-gold nanoparticlulate film surface. b) the ion is complexed in the binding site containing the amine groups (blue), allowing the measurement of its concentration by measuring the changes in the potential. c) Molecular representation of the possible interaction process taking place in the pore cavity of the silica shell between the positively charged amine groups and the chloride ions. The red area indicates the presence of a positively charged environment. 


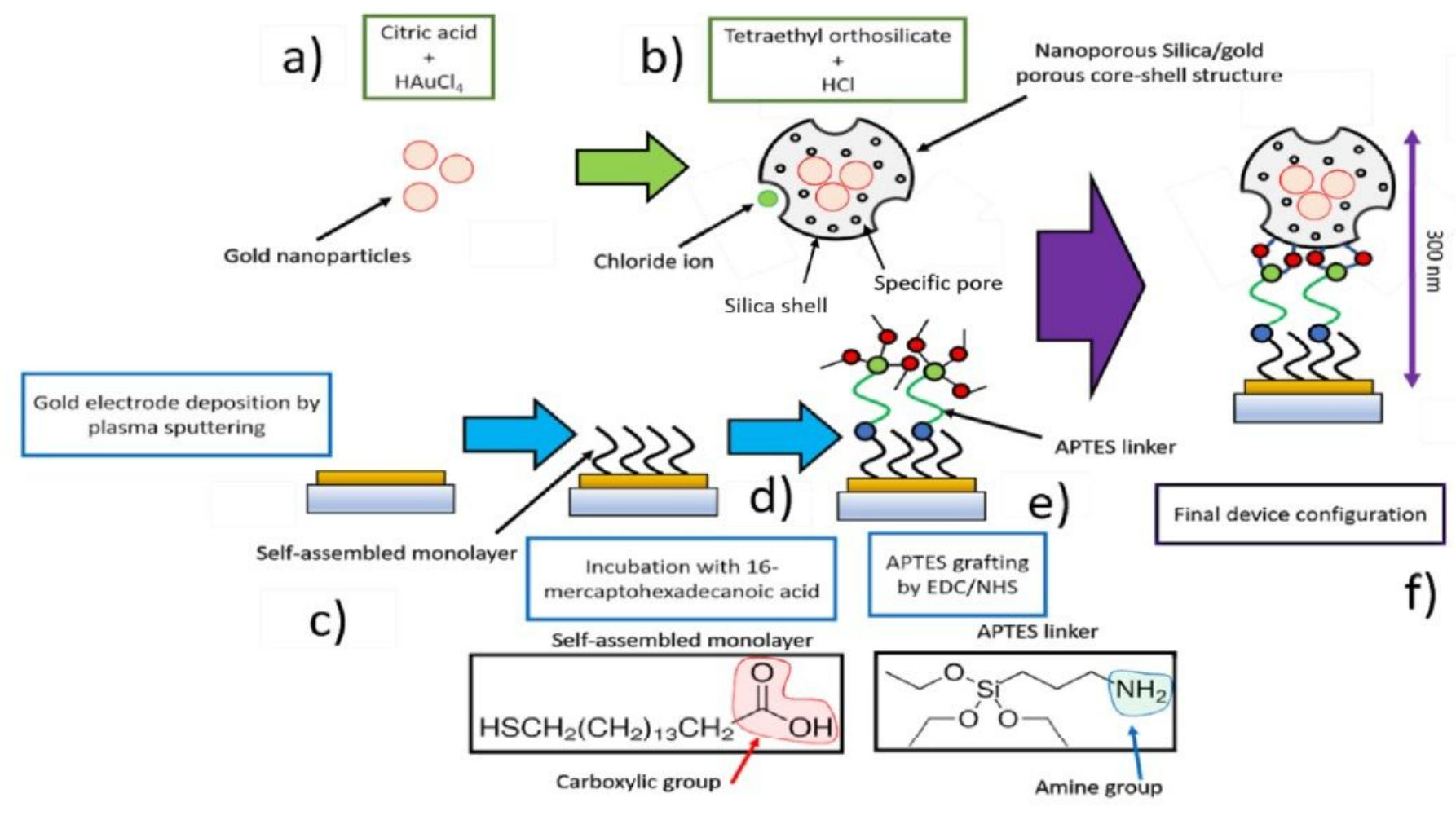

Figure 5

a) Gold NPs synthesis: Au nanoclusters were first synthesized using citric acid as the reducing agent in the presence of HAuCl4. b) Microporous core shell Silica/Au nanoparticles via ionic imprinting method for selective $\mathrm{Cl}$-sensing: The ionically-imprinted imprinted outer silica shell is synthesized using tetraethyl orthosilicate as the structural units and $\mathrm{HCl}$ as the template, using APTES to favor the interaction with the chloride ions. Such APTES is positively charged in the presence of an acid environment as generated by the $\mathrm{HCl}$, increasing the interaction between the porous nanoparticles and the chloride ions. A microporous nanoparticle silica/Au with controlled porosity (circa. $1.5 \mathrm{~nm}$ in pore size) specific towards chloride ions is then developed. c) Electrode fabrication: In parallel, $50 \mathrm{~nm}$ thick gold conducting film was deposited onto glass substrate by plasma sputtering. d) Functionalization of Au electrode: This is achieved by introducing a self-assembled monolayer of 16-mercaptohexadecanoic acid, containing a -SH group that can strongly bind to the gold film, and a carboxyl group(- $\mathrm{COOH})$. e) Linkages: The free-COOH group of the functionalized Au electrode in d) was used to guide the directional attachment of the APTES linker that bridge the electrode with the siloxane groups on the core shell silica-gold synthesized in b) in order to produce f) via the use standard EDC/NHS chemistry; f) A $300 \mathrm{~nm}$ thick nanostructured sensing film is finally produced specific for the detection of chloride ions.

\section{Supplementary Files}

This is a list of supplementary files associated with this preprint. Click to download. 
- SupplementaryMaterials.pdf 